\title{
A Posteriori Dual-Mixed Adaptive Finite Element Error Control for Lamé and Stokes Equations
}

\section{A Posteriori Dual-Mixed AFE Error Control}

\author{
Carsten Carstensen $^{1 *}$, Paola Causin ${ }^{2}$, Riccardo Sacco ${ }^{3}$ \\ 1 Department of Mathematics, Humboldt-Universität zu Berlin, Unter den Linden 6, D- \\ 10099 Berlin, Germany. E-Mail: cc@math. hu-berlin. de \\ 2 INRIA Rocquencourt, B.P.105 Domaine de Voluceau, F-78153 Le Chesnay Cedex, Roc- \\ quencourt, France. E-Mail: paola.causin@inria.fr \\ 3 Dipartimento di Matematica “F. Brioschi”, Politecnico di Milano, via Bonardi 9, 20133 \\ Milano, Italy. E-Mail: riccardo.sacco@mate.polimi.it
}

Version: March 10, 2005

\begin{abstract}
A unified and robust mathematical model for compressible and incompressible linear elasticity can be obtained by rephrasing the Herrmann formulation within the Hellinger-Reissner principle. This quasi-optimally converging extension of PEERS (Plane Elasticity Element with Reduced Symmetry) is called Dual-Mixed Hybrid formulation (DMH). Explicit residual-based a posteriori error estimates for DMH are introduced and are mathematically shown to be locking-free, reliable, and efficient. The estimator serves as a refinement indicator in an adaptive algorithm for effective automatic mesh generation. Numerical evidence supports that the adaptive scheme leads to optimal convergence for Lamé and Stokes benchmark problems with singularities.
\end{abstract}

\section{Introduction and motivation}

It is well known that for nearly incompressible and incompressible materials, i.e. for a value of the Poisson ratio near or equal to 0.5 , finite element computations based on a standard displacement formulation fail due to the onset of the locking phenomenon (see [12] for numerical evidence). A valid alternative to locking-affected methods is represented by dual-mixed

* Supported by the DFG Research Center MATHEON "Mathematics for key technologies" in Berlin 
formulations, that provide mathematical models capable of treating under an unified framework both compressible and incompressible linear elasticity problems (see $[3,21])$. However, the quasi-optimal convergence rate of such methods can be unfavorably degraded, for example, by the presence of singularities in the computational domain. In such an event, the convergence performance can be improved by resorting to a robust meshrefinining algorithm for an efficient automatic mesh-design. A list of contributions proposing and analyzing robust and effective adaptive finite element methods in compressible, nearly incompressible and pure incompressible solid and fluid mechanics includes references $[4,11,12,14,13,20$, $28,29,5,22,23]$.

In the sequel, we will deal with a dual-mixed formulation obtained by rephrasing the Herrmann approach [25] within the Hellinger-Reissner principle. This quasi-optimally converging extension of PEERS (Plane Elasticity Element with Reduced Symmetry) is called Dual-Mixed Hybrid formulation $(\mathrm{DMH})$ and in the case of isotropic materials reads: Given the linear functionals $G(\tau)$ and $F(v)$, find $(\sigma, u, \gamma, p) \in \Sigma_{g_{N}} \times U \times W \times Q$ such that

$$
\begin{array}{ll}
a(\sigma ; \tau)+b(u, \gamma, p ; \tau)=G(\tau) & \forall \tau \in \Sigma_{0}, \\
b(\sigma ; v, \theta, q)+c(p ; q)=F(v) & \forall(v, \theta, q) \in U \times W \times Q .
\end{array}
$$

Therein, $\Sigma_{g_{N}}\left(\right.$ respectively, $\left.\Sigma_{0}\right)$ denotes the Sobolev space $\Sigma=H(\operatorname{div} ; \Omega)$ accounting for nonvanishing (respectively, vanishing) traction boundary conditions on $\Gamma_{N}$, while $U, W$ and $Q$ are Lebesgue spaces over the bounded domain $\Omega \subset \mathbb{R}^{d}$ with Lipschitz boundary $\Gamma=\Gamma_{D} \cup \Gamma_{N}$ for Dirichlet and traction boundary conditions. The bilinear forms $a(\cdot, \cdot), b(\cdot, \cdot)$, and $c(\cdot, \cdot)$ are defined by

$$
\begin{aligned}
& a(\sigma ; \tau)=\frac{1}{2 \mu} \int_{\Omega} \sigma: \tau d x \quad \forall \sigma, \tau \in \Sigma, \\
& b(v, \theta, q ; \tau)=\int_{\Omega} v \cdot \operatorname{div} \tau d x+\int_{\Omega} \theta: \tau d x+\int_{\Omega} \frac{\varrho}{2} q \operatorname{Tr} \tau d x \\
& \forall(v, \theta, q) \in U \times W \times Q \quad \forall \tau \in \Sigma_{0}, \\
& c(p ; q)=\int_{\Omega} \varrho p q d x \quad \forall p, q \in Q,
\end{aligned}
$$

where we set $\sigma: \tau=\sum_{i, j=1}^{d} \sigma_{i j} \tau_{i j}$ and $\operatorname{Tr} \tau=\sum_{i=1}^{d} \tau_{i i}$. The linear forms $G(\tau)$ and $F(v)$ are defined by

$$
G(\tau)=\int_{\Gamma_{D}} u_{D} \cdot \tau n d s \quad \text { and } \quad F(v)=-\int_{\Omega} f \cdot v d x \quad \forall \tau \in \Sigma \forall v \in U .
$$


The parameter $\varrho$ is a continuous function (also in the incompressible limit) of the compressibility modulus $\lambda$ of the material, while $\mu$ is the second elastic Lamé coefficient, We refer the reader to Section 2 for the remaining details of notation.

Quasi-optimal and robust a priori error estimates for DMH were proved in [16]. Robustness here is referred to the performance of the DMH method in the sense that, for small values of the mesh size, the error is independent of the compressibility parameter. As anticipated before, the quasi-optimal convergence rate of $\mathrm{DMH}$ can be degraded by the presence of singularities in the computational domain, this demanding to resort to a robust meshrefinining algorithm for an efficient automatic mesh-design. With this aim, we introduce in this paper explicit residual-based a posteriori error estimates for the DMH formulation. These a posteriori error estimates are used in an adaptive algorithm for effective automatic mesh generation (cf. Section 5).

For $d=2$, we propose the following error estimator with volume contribution $\eta_{K}$ and edge contribution $\eta_{E}$ : Given the computed discrete approximation $\left(\sigma_{h}, u_{h}, p_{h}, \gamma_{h}\right)$, we compute on each element $K$ in the triangulation $\mathcal{T}_{h}$ the volume part of the refinement indicator

$$
\begin{aligned}
\eta_{K}^{2}= & \frac{h_{K}^{2}}{\mu^{2}}\left\|f+\operatorname{div} \sigma_{h}\right\|_{0, K}^{2}+h_{K}^{2}\left\|\operatorname{curl}\left(\frac{1}{2 \mu} \sigma_{h}+\frac{\varrho}{2} p_{h} \delta+\gamma_{h}\right)\right\|_{0, K}^{2} \\
& +\frac{1}{\mu^{2}}\left\|\operatorname{As} \sigma_{h}\right\|_{0, K}^{2}+\left\|\frac{\varrho}{2}\left(p_{h}+\frac{1}{2} \operatorname{Tr} \sigma_{h}\right)\right\|_{0, K}^{2} .
\end{aligned}
$$

Here and throughout the paper, As $\tau=\tau_{12}-\tau_{21}$ for $d=2$, while $\|\cdot\|_{0, S}$ denotes the $L^{2}=H^{0}$ norm $\|\cdot\|_{L^{2}(S)}$ on a domain, face or edge $S$. On each edge $E \in \mathcal{E}_{h}=\mathcal{E}_{\Omega} \cup \mathcal{E}_{D} \cup \mathcal{E}_{N}$, where $\mathcal{E}_{\Omega}$ denotes the set of interior edges, $\mathcal{E}_{D}$ and $\mathcal{E}_{N}$ the sets of edges on the Dirichlet and Neumann boundary, respectively, we compute the jump contribution to the refinement indicator

$$
\eta_{E}^{2}= \begin{cases}h_{E}\left\|J\left(\left(\frac{1}{2 \mu} \sigma_{h}+\frac{\varrho}{2} p_{h} \delta+\gamma_{h}\right) t_{E}\right)\right\|_{0, E}^{2} & \text { if } E \in \mathcal{E}_{\Omega}, \\ h_{E}\left\|\left(\frac{1}{2 \mu} \sigma_{h}+\varrho / 2 p_{h} \delta+\gamma_{h}-\nabla u_{D}\right) t_{E}\right\|_{0, E}^{2} & \text { if } E \in \mathcal{E}_{D}, \\ \frac{h_{E}}{\mu^{2}}\left\|\sigma_{h} n_{E}-g_{N}\right\|_{0, E}^{2} & \text { if } E \in \mathcal{E}_{N} .\end{cases}
$$

The error estimator associated with the DMH formulation, then reads

$$
\Phi\left(\sigma_{h} ; \mathcal{T}_{h}\right)^{2}:=\sum_{K \in \mathcal{T}_{h}} \Phi_{K}^{2} \quad \text { with } \quad \Phi_{K}^{2}:=\eta_{K}^{2}+\sum_{E \subseteq \partial K} \eta_{E}^{2} .
$$

The reliability of the error estimator is expressed by the following result. 
Theorem 1 Let $\mathcal{T}_{h}$ be a regular triangulation of $\Omega$ and let $\left(\sigma_{h}, u_{h}, p_{h}, \gamma_{h}\right)$ be the DMH finite element approximation of the solution of problem (1). Then, there exists a positive constant $C_{\text {rel }}$ independent of the mesh-size $h$ and of the material parameter $\lambda \in[0, \infty]$, such that

$$
\begin{aligned}
& (2 \mu)^{-1 / 2}\left\|\sigma-\sigma_{h}\right\|_{0, \Omega}+(2 \mu)^{1 / 2}\left\|\gamma-\gamma_{h}\right\|_{0, \Omega} \\
& +\left\|p-p_{h}\right\|_{0, \Omega} \leq C_{r e l} \Phi\left(\sigma_{h} ; \mathcal{T}_{h}\right) .
\end{aligned}
$$

The efficiency in a local form of the error estimator is expressed by the following result.

Theorem 2 For each $K \in \mathcal{T}_{h}$, there exists a positive constant $C_{\text {eff, } K}$ independent of the mesh-size $h$ and of the material parameter $\lambda \in[0, \infty]$, such that

$$
\begin{gathered}
\eta_{K} \leq C_{e f f, K}\left(\left\|\sigma-\sigma_{h}\right\|_{0, K}+\left\|p-p_{h}\right\|_{0, K}\right. \\
\left.+\left\|\gamma-\gamma_{h}\right\|_{0, K}+h_{K}\left\|f-f_{K}\right\|_{0, K}\right) .
\end{gathered}
$$

Moreover, for each $E \in \mathcal{E}_{h}$, there exist positive constants $C_{\text {eff, } E_{N}}$ and $C_{e f f, E_{D}}$ independent of the mesh-size $h$ and of the material parameter $\lambda \in$ $[0, \infty]$, such that

$$
\eta_{E} \leq\left\{\begin{array}{c}
C_{e f f, E_{N}}\left(\left\|\sigma-\sigma_{h}\right\|_{0, \omega_{E}}+\left\|h_{E}\left(f-f_{\mathcal{T}_{h}}\right)\right\|_{0, \omega_{E}}\right. \\
\left.+h_{E}^{1 / 2}\left\|g_{N}-g_{N, E}\right\|_{0, E}\right) \quad \forall E \in \mathcal{E}_{N}, \\
C_{e f f, E_{D}}\left(\left\|\sigma-\sigma_{h}\right\|_{0, \omega_{E}}+\left\|p-p_{h}\right\|_{0, \omega_{E}}\right. \\
\left.+\left\|\gamma-\gamma_{h}\right\|_{0, \omega_{E}}+h_{E}^{1 / 2}\left|u_{D}-u_{D, E}\right|_{1, E}\right) \quad \forall E \in \mathcal{E}_{D},
\end{array}\right.
$$

where for the two neighbouring elements $K=T_{ \pm}, \bar{\omega}_{E}=T_{+} \cup T_{-}$and $T_{ \pm} \in \mathcal{T}_{h}$ with $T_{+} \cap T_{-}=E$.

Details on the aformentioned notation are given in Sects. 2 and 3. The proof of Thm. 1 combines a unified approach from [10] with several arguments from $[11,12,14,13,7]$. Efficiency holds in a local form up to higherorder terms.

The local refinement indicator $\Phi_{K}$ from (3) serves in an adaptive algorithm for effective automatic mesh generation. Numerical evidence in Section 6 supports that the resulting DMH adaptive scheme is characterized by optimal convergence rates when applied to the numerical solution of Lamé and Stokes benchmark problems with singularities.

The remaining part of the paper is organized as follows. Section 2 is devoted to the strong form of the linear elasticity problem and its related dual-mixed weak formulation. The numerical approximation of the DMH problem is then considered, and a priori quasi-optimal error estimates are reviewed for the method, with emphasis on their robustness with respect to the compressibility parameter. Section 2 concludes with some details on the 
efficient implementation of the dual-mixed method through the hybridization procedure; cf. $[1,2,16]$. A proof of the a posteriori error estimate (4) is given in Section 3 while efficiency of $\Phi\left(\sigma_{h} ; \mathcal{T}_{h}\right)$ is discussed in Section 4. Section 5 illustrates the adaptive algorithm implemented in the computer code to drive the automatic mesh generation process. Numerical experiments on several benchmark problems illustrate in Section 6 the reliability and the efficiency of our robust a posteriori error estimate as well as the performance of the proposed adative refinement strategy.

\section{Model problem, dual-mixed formulation, and finite element approximation}

\subsection{Mathematical model}

Let $\Omega \subset \mathbb{R}^{d}$ be the reference configuration of an elastic material with Lipschitz boundary $\partial \Omega=\Gamma_{D} \cup \Gamma_{N}, \Gamma_{D} \neq \emptyset, \Gamma_{D} \cap \Gamma_{N}=\emptyset$, and its outward unit normal vector $n$. The linear elasticity problem reads: Given a volume force $f: \Omega \rightarrow \mathbb{R}^{d}$, a displacement $u_{D}: \Gamma_{D} \rightarrow \mathbb{R}^{d}$, a traction $g_{N}: \Gamma_{N} \rightarrow \mathbb{R}^{d}$, find a displacement $u: \Omega \rightarrow \mathbb{R}^{d}$ and a stress $\sigma: \Omega \rightarrow M_{\text {sym }}^{d \times d}=\left\{\tau \in \mathbb{R}^{d \times d}: \tau=\tau^{T}\right\}$ satisfying

$$
\begin{aligned}
-\operatorname{div} \sigma & =f & & \text { in } \Omega, \\
\sigma & =\mathbb{C} \varepsilon(u) & & \text { in } \Omega, \\
u & =u_{D} & & \text { on } \Gamma_{D}, \\
\sigma n & =g_{N} & & \text { on } \Gamma_{N}
\end{aligned}
$$

with strain tensor $\varepsilon(v)=\frac{1}{2}\left(\nabla v+(\nabla v)^{T}\right)$ and stress tensor

$$
\sigma=\mathbb{C} \epsilon(u)=2 \mu \epsilon(u)+\lambda \operatorname{Tr} \epsilon(u) \delta
$$

in the isotropic case. In (8), $\lambda, \mu$ are the Lamé constants and $\delta$ is the $d \times d$ identity matrix. Plain strain or plane stress conditions can be recovered by taking in (7) appropriate values of the coefficients $\lambda$ and $\mu$ (see [26], p.83).

Korn's inequality and Lax-Milgram lemma ensure that problem (7) admits a unique (weak) solution $(\sigma, u) \in L^{2}\left(\Omega ; M_{\text {sym }}^{d \times d}\right) \times H^{1}(\Omega)^{d}$. In order to construct the DMH variational formulation of (7) we introduce two additional unknowns, $p$ and $\gamma$, through the relations

$$
p=-\frac{1}{d} \operatorname{Tr} \sigma \quad \text { and } \quad \varepsilon(u)=\nabla u-\gamma .
$$

The variable $p$ is a pressure parameter that allows for a straightforward discrete treatment of the incompressible case $(\lambda=+\infty)$ and is the main novelty of the DMH formulation with respect to the original PEERS approach [2]. Denoting by $\bar{p}$ the hydrostatic pressure, in the case $d=3$ we have that $p=\bar{p}$, while when $d=2, p=((\lambda+\mu) /(\lambda+2 \mu / 3)) \bar{p}$ in the 
case of plain strain conditions and $p=3 \bar{p} / 2$ in the case of plane stress conditions. The variable $\gamma$ is the infinitesimal rotation tensor that allows to weaken the symmetry constraint on $\sigma$.

Introducing (9) into (7), we obtain the following equivalent formulation of the linear elasticity problem [16]: Find $u: \Omega \rightarrow \mathbb{R}^{d}, \sigma: \Omega \rightarrow M^{d \times d}$, $p: \Omega \rightarrow \mathbb{R}$, and $\gamma: \Omega \rightarrow M_{\text {skew }}^{d \times d}=\left\{\eta \in M^{d \times d}: \eta+\eta^{T}=0\right\}$ such that

$$
\begin{aligned}
-\operatorname{div} \sigma & =f & & \text { in } \Omega, \\
\sigma & =\widetilde{\mathbb{C}}(\nabla u-\gamma)-\mu \varrho p \delta & & \text { in } \Omega, \\
p & =-\frac{1}{d} \operatorname{Tr} \sigma & & \text { in } \Omega, \\
\operatorname{As} \sigma & =0 & & \text { in } \Omega, \\
u & =u_{D} & & \text { on } \Gamma_{D}, \\
\sigma n & =g_{N} & & \text { on } \Gamma_{N}
\end{aligned}
$$

where $\widetilde{\mathbb{C}}=2 \mu$ and $\varrho=\lambda /(\mu(\lambda+2 \mu / d))$, with $\lim _{\lambda \rightarrow \infty} \varrho=1 / \mu$. In the incompressible case, system (10) can be conveniently interpreted as the conservative form of the Stokes equations in fluid dynamics

$$
\begin{aligned}
-\operatorname{div} \sigma(u) & =f & & \text { in } \Omega, \\
\sigma & =2 \nu \epsilon(u)-p \delta & & \text { in } \Omega, \\
\operatorname{div} u & =0 & & \text { in } \Omega, \\
u & =u_{D} & & \text { on } \Gamma_{D}, \\
\sigma(u) n & =g_{N} & & \text { on } \Gamma_{N}
\end{aligned}
$$

with kinematic viscosity $\nu=\mu$ and where $u$ is to be intended as a velocity field. To show the equivalence of system (11) with the Stokes problem in conservative form, we use the definition of $\gamma$ in $(10)_{2}$, take the trace of this latter relation, and use the definition of $\widetilde{\mathbb{C}}$ and $p$, obtaining

$$
\operatorname{Tr} \epsilon(u)=\operatorname{div} u=\frac{1}{2 \mu}(\operatorname{Tr} \sigma+\mu \varrho p d)=\frac{p d}{2 \mu}(\mu \varrho-1) .
$$

For $\lambda=+\infty$, the incompressibility constraint $\operatorname{div} u=0$ is thus recovered.

\subsection{DMH weak formulation}

In order to introduce the DMH weak formulation of problem (10), we define the Sobolev space

$$
H(\operatorname{div} ; \Omega)=\left\{\sigma \in L^{2}\left(\Omega ; M^{d \times d}\right): \operatorname{div} \sigma \in L^{2}(\Omega)^{d}\right\} ;
$$

and, given $u_{D} \in L^{2}\left(\Gamma_{D}\right)^{d}, g_{N} \in L^{2}\left(\Gamma_{N}\right)^{d}$ and $f \in L^{2}(\Omega)^{d}$,

$$
\begin{aligned}
& \Sigma_{g_{N}}=\left\{\sigma \in H(\operatorname{div} ; \Omega): \sigma n=g_{N} \text { on } \Gamma_{N}\right\}, \\
& U=L^{2}(\Omega)^{d}, \quad W=L^{2}\left(\Omega ; M_{\text {skew }}^{d \times d}\right), \quad Q=L^{2}(\Omega) .
\end{aligned}
$$


In the case $\Gamma_{N}=\emptyset$, in order to preserve the uniqueness of the solution, the definition of the pressure space $Q$ must be modified into $Q=L_{0}^{2}(\Omega)$, the space of square integrable functions over $\Omega$ having null average on $\Omega$. Multiplying each equation in (10) by a proper test function and using integration by parts, we obtain the DMH weak formulation (1) of the linear elasticity problem. Under proper regularity assumptions on the solution of (10), an existence and uniqueness result of the solution of (1) has been proved in [16].

\subsection{Finite element approximation}

In view of the numerical approximation of problem (1) we assume henceforth that $\Omega$ is a bounded domain in $\mathbb{R}^{2}$ and we introduce a regular partition $\mathcal{T}_{h}$ [17] of $\Omega$ into triangles $K$ such that $\bar{\Omega}=\bigcup_{K \in \mathcal{T}_{h}} \bar{K}$ and let $\mathcal{E}_{h}=\mathcal{E}_{\Omega} \cup \mathcal{E}_{D} \cup \mathcal{E}_{N}$ be the set of edges associated with $\mathcal{T}_{h}$. For each element $K \in \mathcal{T}_{h}$ with boundary $\partial K$, we denote by $|K|$ and $h_{K}$ the area and the diameter of $K$, respectively, while for each edge $E \in \mathcal{E}_{h}$ we denote by $h_{E}$ the length of $E$ and choose one unit normal $n_{E}$ along $E$ (pointing outwards $\Omega$ for $E \in \mathcal{E}_{N}$ ). We set $h=\max _{K \in \mathcal{T}_{h}} h_{K}$. Then, for $k \geq 0$, we denote by $\mathbb{P}_{k}(K)$ the space of polynomials in two variables of total degree at most $k$ on the element $K$ and we let $\mathbb{D}(K)=\left(\mathbb{R T}_{0}(K) \oplus \mathcal{B}_{K}\right)^{2}$, where $\mathbb{R T}_{0}(K)$ is the lowest order Raviart-Thomas finite element space [27] on $K$ and $\mathcal{B}_{K}=\operatorname{curl}\left(b_{K}\right), b_{K}$ being the cubic bubble function on $K$ [9]. The finite element spaces for the DMH approximation are defined as follows

$$
\begin{aligned}
\Sigma_{g_{N}, h}= & \left\{\sigma_{h} \in H(\operatorname{div} ; \Omega): \forall E \in \mathcal{E}_{N},\left.\sigma_{h} n\right|_{E}=\mathcal{P}_{0, E}\left(g_{N}\right) ;\right. \\
& \left.\forall K \in \mathcal{T}_{h},\left.\sigma_{h}\right|_{K} \in \mathbb{D}(K)\right\}, \\
U_{h}= & \left\{u_{h} \in U: \forall K \in \mathcal{T}_{h},\left.u_{h}\right|_{K} \in\left(\mathbb{P}_{0}(K)\right)^{2}\right\}, \\
W_{h}= & \left\{\gamma_{h} \in C^{0}\left(\bar{\Omega} ; M_{\text {skew }}^{2 \times 2}\right): \forall K \in \mathcal{T}_{h},\left.\gamma_{h}\right|_{K} \in \mathbb{P}_{1}\left(K ; M_{\text {skew }}^{2 \times 2}\right)\right\}, \\
Q_{h}= & \left\{q_{h} \in Q: \forall K \in \mathcal{T}_{h},\left.q_{h}\right|_{K} \in \mathbb{P}_{0}(K)\right\} .
\end{aligned}
$$

Therein, for any $E \in \mathcal{E}_{h}, \mathcal{P}_{0, E}$ is the orthogonal projection in $L^{2}(E)$ onto the space of constants $\left(\mathbb{P}_{0}(E)\right)^{2}$ on $E$, i.e. $\mathcal{P}_{0, E}$ is the integral norm operator.

The discretization of problem (1) reads: Find $\left(\sigma_{h}, u_{h}, \gamma_{h}, p_{h}\right) \in \Sigma_{g_{N}, h} \times$ $U_{h} \times W_{h} \times Q_{h}$ such that

$$
\begin{array}{ll}
a\left(\sigma_{h} ; \tau_{h}\right)+b\left(u_{h}, \gamma_{h}, p_{h} ; \tau_{h}\right)=G\left(\tau_{h}\right) & \forall \tau_{h} \in \Sigma_{0, h}, \\
b\left(\sigma_{h} ; v_{h}, \theta_{h}, q_{h}\right)+c\left(p_{h} ; q_{h}\right)=F\left(v_{h}\right) & \forall\left(v_{h}, \theta_{h}, q_{h}\right) \in U_{h} \times W_{h} \times Q_{h} .
\end{array}
$$


Existence and uniqueness of the solution of the discretized problem (13) have been proved in [16] and in the same reference, under appropriate (minimal) regularity assumptions on the solution of problem (1), the following quasi-optimal a priori error estimates have been established

$$
\begin{aligned}
& \left\|\sigma-\sigma_{h}\right\|_{0, \Omega}+\| p-\left.p_{h}\right|_{0, \Omega} \lesssim h\left(|\sigma|_{1, \Omega}+|p|_{1, \Omega}+|\gamma|_{1, \Omega}\right), \\
& \left\|u-u_{h}\right\|_{0, \Omega} \lesssim h\left(|\sigma|_{1, \Omega}+|p|_{1, \Omega}+|u|_{1, \Omega}+|\gamma|_{1, \Omega}\right) \\
& \left\|\gamma-\gamma_{h}\right\|_{0, \Omega} \lesssim h\left(|\sigma|_{1, \Omega}+|p|_{1, \Omega}+|\gamma|_{1, \Omega}\right) .
\end{aligned}
$$

Here and throughout this paper, $A \leq C B$ is abbreviated as $A \lesssim B$ when $C$ is a positive (generic) constant independent of $h$ and independent of the compressibility parameter $\lambda$; for any integer $m \geq 0$ we denote by $\|\cdot\|_{m, S}$ and $|\cdot|_{m, S}$ the norm and seminorm on the Sobolev space $H^{m}(S)$, respectively, $S \subset \mathbb{R}^{d}$. It is important to notice that the above estimates are uniformly robust with respect to the compressibility parameter $\lambda$, i.e. they maintain their validity even in the exactly incompressible regime characterized by the value $\lambda=+\infty$.

Remark 1 The computer implementation of (13) leads to solving a linear sparse system of algebraic equations of large size. In order to reduce the computational complexity of the problem, it is convenient to resort to the hybridization of the dual-mixed formulation (13). This amounts to relaxing the $H(\operatorname{div} ; \Omega)$-continuity requirement for interelement normal stresses that is contained in the definition of the finite element space $\Sigma_{g_{N}, h}$ at the expense of introducing a further variable $\lambda_{h}$ into the system (13) (see [1,2] and [19]). The variable $\lambda_{h}$ is defined only over the edges of $\mathcal{E}_{h}$ and plays the role of a Lagrangian multiplier that enforces back the interelement traction reciprocity of the discrete stress $\sigma_{h}$. The resulting discontinuous finite element spaces for $\sigma_{h}$ and $\lambda_{h}$ are defined as

$$
\begin{aligned}
& \Sigma_{h}=\left\{\sigma_{h} \in L^{2}\left(\Omega ; M^{2 \times 2}\right): \forall K \in \mathcal{T}_{h},\left.\sigma_{h}\right|_{K} \in \mathbb{D}(K)\right\}, \\
& \Lambda_{u_{D}, h}=\left\{\lambda_{h} \in L^{2}\left(\mathcal{E}_{h}\right)^{2}: \forall E \in \mathcal{E}_{h},\left.\lambda_{h}\right|_{E} \in\left(\mathbb{P}_{0}(E)\right)^{2},\right. \\
& \\
&\left.\forall E \in \mathcal{E}_{D},\left.\lambda_{h}\right|_{E}=\mathcal{P}_{0, E} u_{D}\right\} .
\end{aligned}
$$

Since the stress $\sigma_{h}$ is now a priori fully discontinuous over the triangulation $\mathcal{T}_{h}$, it can be statically eliminated at the element level together with the element displacement $u_{h}$, leading to a condensed system in the sole unknowns $\gamma_{h}, p_{h}, \lambda_{h}$ (see $[12,15]$ for the algorithmic details of the procedure). The interelement variable $\lambda_{h}$ has the physical meaning of edge displacement and can be shown to enjoy a higher convergence rate than the corresponding element variable $u_{h}$ (see [16] and also [1,21] and [18]). 


\section{A posteriori error estimate}

This section is devoted to prove Theorem 1. Beforehand, we need to introduce some notation that will be useful in the following.

\subsection{Notation}

For each edge $E \in \mathcal{E}_{h}$ with fixed normal $n_{E}$ (that coincides with the exterior normal to $\Gamma$ if $E \subset \Gamma$ ), define the jump of a function $v$ (that is continuous on each of the neighbouring elements $K_{+}$and $K_{-}$but, in general, discontinuous along their intersection $E$ ) across the edge $E$ as

$$
\left.J(v)\right|_{E}:=\left(\left.\left.v\right|_{K_{+}}\right|_{E}\right)-\left(\left.\left.v\right|_{K_{-}}\right|_{E}\right),
$$

$K^{+}$being the triangle whose outward unit normal vector coincides with $n_{E}$. If $E=K \cap \Gamma$, we set

$$
\left.J(v)\right|_{E}:=\left(\left.\left.v\right|_{K}\right|_{E}\right) .
$$

For each normal $n_{E}$ we denote by $t_{E}$ its associated tangent unit vector, such that $n_{E}$ points to the right side defined by the orientation of $t_{E}$.

Moreover, for all $\phi \in H^{1}(\Omega), u \in H^{1}(\Omega)^{2}$ and $\sigma \in H^{1}\left(\Omega ; M^{2 \times 2}\right)$, we define

$$
\begin{array}{lll}
\operatorname{Curl} \phi & =\left(\phi_{, 2}-\phi_{, 1}\right), & \operatorname{curl} \phi=\left(\begin{array}{c}
\phi_{, 2} \\
-\phi_{, 1}
\end{array}\right) ; \\
\operatorname{Curl} u=\left(\begin{array}{l}
u_{1,2}-u_{1,1} \\
u_{2,2}-u_{2,1}
\end{array}\right), & \operatorname{curl} u=u_{2,1}-u_{1,2} ; \\
\operatorname{curl} \sigma=\left(\begin{array}{l}
\sigma_{12,1}-\sigma_{11,2} \\
\sigma_{22,1}-\sigma_{21,2}
\end{array}\right), & \operatorname{div} \sigma=\left(\begin{array}{c}
\sigma_{11,1}+\sigma_{12,2} \\
\sigma_{21,1}+\sigma_{22,2}
\end{array}\right) .
\end{array}
$$

In the sequel, the mesh-size is regarded as a (piecewise constant) $L^{\infty}$ function, i.e. $h_{\mathcal{T}_{h}}$ (respectively, $h_{\mathcal{E}_{h}}$ ) is $\mathcal{T}_{h}$-piecewise constant (respectively, $\mathcal{E}_{h^{-}}$ piecewise constant) with $\left.h_{\mathcal{T}_{h}}\right|_{K}=h_{K}=\operatorname{diam}(K)$ for each $K \in \mathcal{T}_{h}$ (respectively $\left.h_{\mathcal{E}_{h}}\right|_{E}=h_{E}=\operatorname{diam}(E)$ for each $E \in \mathcal{E}_{h}$ ). Similarly, the piecewise action of differential operators on (in general discontinuous) piecewise sufficiently smooth functions is denoted by a subindex, e.g. Curl $\mathcal{T}_{h}$ means

$$
\left.\left(\operatorname{Curl}_{\mathcal{T}_{h}} v\right)\right|_{K}=\operatorname{Curl}\left(\left.v\right|_{K}\right) \quad \text { for } v \in H^{1}(K), \quad \forall K \in \mathcal{T}_{h} .
$$

For brevity, let $(\cdot, \cdot)_{0, S}$ denote the $L^{2}$ scalar product in $L^{2}(S)$ for a domain, edge, etc., and let $\|\cdot\|_{0, S}$ denote the induced norm. Similarly, $\|\cdot\|_{1, S}$ and $\mid$. $\left.\right|_{1, S}$ denote the norm and seminorm in $H^{1}(S)$, respectively. Set $H_{D}^{1}(\Omega):=$ 
$\left\{v \in H^{1}(\Omega)^{2}: v=0\right.$ on $\left.\Gamma_{D}\right\}$ and $H_{0}^{1}(\Omega):=\left\{v \in H^{1}(\Omega)^{2}: v=\right.$ 0 on $\Gamma\}$ and

$L:=\left\{\sigma \in L^{2}\left(\Omega ; M_{\mathrm{sym}}^{2 \times 2}\right): \int_{\Omega} \operatorname{Tr}(\sigma) d x=0\right\}$ and $V:=H_{0}^{1}(\Omega)^{2}$ if $\Gamma_{N}=\emptyset ;$

$L:=L^{2}\left(\Omega ; M_{\mathrm{sym}}^{2 \times 2}\right)$ and $V:=H_{D}^{1}(\Omega)$ if $\Gamma_{N} \neq \emptyset$.

Let $\|\cdot\|_{L}:=\|\cdot\|_{0, \Omega}$ and $\|\cdot\|_{V}:=\|\cdot\|_{1, \Omega}$. With the elasticity tensor $\mathbb{C}$ from (8) and the positive parameters $\mu$ and $\lambda$ one defines the energy norms

$$
\|\varepsilon(v)\|_{\mathbb{C}}:=(\mathbb{C} \varepsilon(v), \varepsilon(v))_{0, \Omega}^{1 / 2} \quad \text { and } \quad\|\tau\|_{\mathbb{C}^{-1}}:=\left(\mathbb{C}^{-1} \tau, \tau\right)_{0, \Omega}^{1 / 2}
$$

for any $\varepsilon(v)$ or $\tau$ in $L^{2}\left(\Omega ; M^{2 \times 2}\right)$.

\subsection{Mathematical preliminaries}

Given the exact and discrete solution $(\sigma, u, \gamma, p)$ and $\left(\sigma_{h}, u_{h}, \gamma_{h}, p_{h}\right)$ in $H(\operatorname{div} ; \Omega) \times U \times W \times Q$, define $\tilde{\sigma}_{h}:=\operatorname{sym} \sigma_{h}$, the symmetric part of $\sigma_{h}$ and let $w \in H^{1}(\Omega)^{2}$ be some Sobolev function with $w=u_{D}$ on $\Gamma_{D}$.

Since, $\tilde{\sigma}_{h}$ does, in general, not equal $\mathbb{C} \varepsilon\left(\tilde{u}_{h}\right)$ for some $\tilde{u}_{h} \in H_{D}^{1}(\Omega)$, we consider $\tilde{u}_{h}+w$ with the minimal distance with respect to $\mathbb{C} \varepsilon\left(\tilde{u}_{h}\right)$ as defined by the following Helmholtz decomposition.

Lemma 1 (Helmholtz decomposition for symmetric tensor fields, [11]) Suppose that $\Gamma_{N}$ is a finite union of connected components $\Gamma_{0}, \ldots, \Gamma_{M}$ and either $\Gamma_{N}=\emptyset$ or $\Gamma_{N} \neq \emptyset$ and $\Gamma_{D}$ have a positive distance. Then there exists $\tilde{u}_{h} \in H_{D}^{1}(\Omega)$ and $\Phi \in H^{2}(\Omega)$ with $\int_{\Omega} \Phi d x=0, \operatorname{Curl} \Phi=c_{j}$ on $\Gamma_{j}, c_{j} \in \mathbb{R}^{2}, j=1, \ldots, M, c_{0}=0$, such that

$$
\tilde{\sigma}_{h}-\mathbb{C} \varepsilon(w)=\mathbb{C} \varepsilon\left(\tilde{u}_{h}\right)+\operatorname{Curl} \operatorname{Curl} \Phi .
$$

Lemma 2 ([11]) There exists some $\lambda$-independent constant $C_{1}$ (which depends on $\Gamma_{N}, \Omega$, and $\mu$ but not on $\lambda$ or on $\Phi$ ) such that

$$
\|\Phi\|_{2, \Omega}=\|\operatorname{Curl} \operatorname{Curl} \Phi\|_{0, \Omega} \leq C_{1}\|\operatorname{Curl} \operatorname{Curl} \Phi\|_{\mathbb{C}^{-1}} .
$$

Theorem 3 Let $\mathcal{A}: L \times V \rightarrow(L \times V)^{\prime}$ be defined by

$$
(\mathcal{A}(\sigma, u))(\tau, v):=\left(\mathbb{C}^{-1} \sigma, \tau\right)_{0, \Omega}-(\sigma, \varepsilon(v))_{0, \Omega}-(\tau, \varepsilon(u))_{0, \Omega}
$$

for all $\sigma, \tau \in L$ and $u, v \in V$. Then, the operator $\mathcal{A}$ is bounded and bijective and the operator norms of $\mathcal{A}$ and $\mathcal{A}^{-1}$ are $\lambda$-independent. 
Proof. The case $\Gamma_{N}=\emptyset$ is proved in [7, Theorem 5.1] and the proof in the case $\Gamma_{N} \neq \emptyset$ is sketched in the sequel for convenient reading. We refer the reader to $[6,9]$ for the general theory of mixed formulations. The continuity and inf-sup condition on the bilinear form $(\sigma, \varepsilon(u))$ (with $\lambda$ independent constants) are well established. The kernel of this bilinear form reads

$$
Z:=\left\{\sigma \in L: \operatorname{div} \sigma=0 \text { in } \Omega \text { and } \sigma n=0 \text { on } \Gamma_{N}\right\} .
$$

The remaining non-trivial point is to verify that the bilinear form $\left(\mathbb{C}^{-1} \sigma, \tau\right)$ is continuous and $Z$-elliptic with $\lambda$-independent constants. The first property is immediate from the expression of $\mathbb{C}^{-1}$. To check the latter property, given $\sigma \in Z$, we employ the arguments of Lemma 1 and obtain

$$
\sigma=\mathbb{C} \varepsilon(v)+\operatorname{Curl} \operatorname{Curl} \Phi
$$

for $v \in H_{D}^{1}(\Omega)$ and $\Phi$ with properties stated in the lemma. Since $\operatorname{div} \sigma=$ 0 in $\Omega$ and $\sigma n=0=\operatorname{Curl} \operatorname{Curl} \Phi n$ on $\Gamma_{N}$ we deduce $v=0$. From Lemma 4.2 in [11] we have

$$
\|\operatorname{Curl} \operatorname{Curl} \Phi\|_{0, \Omega} \leq C_{1}\|\operatorname{Curl} \operatorname{Curl} \Phi\|_{\mathbb{C}^{-1}}
$$

for a $\lambda$-independent constant $C_{1}$ (which depends on $\Gamma_{N}, \Omega$, and $\mu$ but not on $\Phi)$. Since $\sigma=\operatorname{Curl} \operatorname{Curl} \Phi$, the last inequality reads $C_{1}^{-2}\|\sigma\|_{L}^{2} \leq$ $\left(\mathbb{C}^{-1} \sigma, \sigma\right)$. For more details cf. [7].

\subsection{Proof of reliability.}

Recall that $w \in H^{1}(\Omega)^{2}$ denotes a function with $w=u_{D}$ on $\Gamma_{D}$ and recall from (16), that

$$
\sigma-\tilde{\sigma}_{h}=\mathbb{C} \varepsilon\left(u-w-\tilde{u}_{h}\right)-\operatorname{Curl} \operatorname{Curl} \Phi .
$$

Theorem 3 is then applied to $\left(\sigma-\tilde{\sigma}_{h}, u-w-\tilde{u}_{h}\right)$ and yields existence of $(\tau, v) \in L \times V$ with $\|(\tau, v)\|_{L \times V} \lesssim 1$ uniformly bounded such that

$$
\begin{aligned}
\left\|\sigma-\tilde{\sigma}_{h}\right\|_{L}+\left\|u-w-\tilde{u}_{h}\right\|_{V} \\
=\left(\mathcal{A}\left(\sigma-\tilde{\sigma}_{h}, u-w-\tilde{u}_{h}\right)\right)(\tau, v) \\
=\left(\mathbb{C}^{-1}\left(\sigma-\tilde{\sigma}_{h}\right), \tau\right)_{0, \Omega}-\left(\sigma-\tilde{\sigma}_{h}, \varepsilon(v)\right)_{0, \Omega} \\
\quad-\left(\tau, \varepsilon\left(u-w-\tilde{u}_{h}\right)\right)_{0, \Omega} \\
=-\left(\mathbb{C}^{-1}(\operatorname{Curl} \operatorname{Curl} \Phi), \tau\right)_{0, \Omega}-(\sigma, \varepsilon(v))_{0, \Omega}+\left(\sigma_{h}, \varepsilon(v)\right)_{0, \Omega}
\end{aligned}
$$

because of $\mathbb{C}^{-1} \sigma=\varepsilon(u)$ and $\tilde{\sigma}_{h}: \varepsilon(v)=\sigma_{h}: \varepsilon(v)$.

In the next steps, we estimate the right-hand side of (17). 
Using $(\sigma, \varepsilon(v))_{0, \Omega}=(f, v)_{0, \Omega}+\int_{\Gamma_{N}} g_{N} v d s$ and an integration by parts in the term $\left(\sigma_{h}, \nabla v\right)_{0, \Omega}$ we obtain

$$
\begin{aligned}
\left\|\sigma-\tilde{\sigma}_{h}\right\|_{L}+\left\|u-w-\tilde{u}_{h}\right\|_{V}=( & \left.-\mathbb{C}^{-1}(\operatorname{Curl} \operatorname{Curl} \Phi), \tau\right)_{0, \Omega}-\left(\operatorname{div} \sigma_{h}+f, v\right)_{0, \Omega} \\
& +\left(\sigma_{h}, \varepsilon(v)-\nabla v\right)_{0, \Omega}+\int_{\Gamma_{N}}\left(\sigma_{h} n-g_{N}\right) v d s .
\end{aligned}
$$

Since $\int_{K}\left(f+\operatorname{div} \sigma_{h}\right) d x=0$ for any $K \in \mathcal{T}_{h}$ we have, with $v_{h} \in$ $\left(\mathbb{P}_{0}\left(\mathcal{T}_{h}\right)\right)^{2}$ defined by $\left.v_{h}\right|_{K}:=|K|^{-1} \int_{K} v(x) d x$ for all $K \in \mathcal{T}_{h}$, that

$$
\begin{aligned}
-\left(\operatorname{div} \sigma_{h}+f, v\right)_{0, \Omega} & =-\left(\operatorname{div} \sigma_{h}+f, v-v_{h}\right)_{0, \Omega} \\
& \leq\left\|h_{\mathcal{T}_{h}}\left(f+\operatorname{div} \sigma_{h}\right)\right\|_{0, \Omega}\left\|h_{\mathcal{T}_{h}}^{-1}\left(v-v_{h}\right)\right\|_{0, \Omega} \\
& \lesssim\left\|h_{\mathcal{T}_{h}}\left(f+\operatorname{div} \sigma_{h}\right)\right\|_{0, \Omega}
\end{aligned}
$$

(owing to an elementwise Poincare inequality $\left\|h_{\mathcal{T}_{h}}^{-1}\left(v-v_{h}\right)\right\|_{0, \Omega} \leq 1 / \pi\|\nabla v\|_{0, \Omega}$ $\leq 1)$. Since $\left.\sigma_{h} n\right|_{E}=\mathcal{P}_{0, E} g_{N}$ for each $E \in \mathcal{E}_{N}$, a similar argument shows that

$$
\begin{aligned}
\int_{\Gamma_{N}}\left(\sigma_{h} n-g_{N}\right) v d s & =\int_{\Gamma_{N}}\left(\sigma_{h} n-g_{N}\right)\left(v-v_{\mathcal{E}_{h}}\right) d x \\
& \leq\left\|h_{\mathcal{E}_{h}}^{1 / 2}\left(g_{N}-\sigma_{h} n\right)\right\|_{0, \Gamma_{N}}\left\|h_{\mathcal{E}_{h}}^{-1 / 2}\left(v-v_{\mathcal{\varepsilon}}\right)\right\|_{0, \Gamma_{N}} \\
& \lesssim\left\|h_{\mathcal{E}_{h}}^{1 / 2}\left(g_{N}-\sigma_{h} n\right)\right\|_{0, \Gamma_{N}}
\end{aligned}
$$

(owing to an elementwise trace inequality and a proper choice of an edgewise $\left.v_{\mathcal{E}_{h}}\right)$.

Since $\left(\sigma_{h}, \varepsilon(v)-\nabla v\right)_{0, \Omega}=\left(\operatorname{As} \sigma_{h}, \frac{1}{2} \operatorname{curl} v\right)_{0, \Omega}$, this term is bounded by $\left\|\operatorname{As} \sigma_{h}\right\|_{0, \Omega}\|\varepsilon(v)-\nabla v\|_{0, \Omega} \lesssim\left\|\operatorname{As} \sigma_{h}\right\|_{0, \Omega}$ (where we employed Korn's inequality and $\|v\|_{V} \leq 1$ in the last step).

Altogether, we deduce

$$
\begin{aligned}
\left\|\sigma-\tilde{\sigma}_{h}\right\|_{L}+\left\|u-w-\tilde{u}_{h}\right\|_{V} & \lesssim\|\operatorname{Curl} \operatorname{Curl} \Phi\|_{L}+\left\|h_{\mathcal{T}_{h}}\left(f+\operatorname{div} \mathcal{T}_{h} \sigma_{h}\right)\right\|_{0, \Omega} \\
& +\left\|h_{\mathcal{E}_{h}}^{1 / 2}\left(g_{N}-\sigma_{h} n\right)\right\|_{0, \Gamma_{N}}+\left\|\operatorname{As} \sigma_{h}\right\|_{L} .
\end{aligned}
$$

Lemma 4 There exists a constant $C_{2}>0$ independent of $\lambda$ and $h$, such that

$$
\begin{aligned}
\|\operatorname{Curl} \operatorname{Curl} \Phi\|_{\mathbb{C}^{-1}} \leq & C_{2}\left(\left\|h_{\mathcal{T}_{h}} \operatorname{Curl}_{\mathcal{T}_{h}}\left(\tilde{\mathbb{C}}^{-1} \sigma_{h}+\varrho / 2 p_{h} \delta+\gamma_{h}\right)\right\|_{0, \Omega}\right. \\
& +\left\|\frac{\varrho}{2}\left(p_{h}+\frac{1}{2} \operatorname{Tr} \sigma_{h}\right)\right\|_{0, \Omega} \\
& \left.+\left\|h_{\mathcal{E}_{h}}^{1 / 2} J\left(\tilde{\mathbb{C}}^{-1} \sigma_{h}+\varrho / 2 p_{h} \delta+\gamma_{h}\right) t_{E}\right\|_{0,\left(\mathcal{E}_{\Omega} \cup \Gamma_{D}\right)}\right) .
\end{aligned}
$$


Proof. Notice that

$$
\begin{aligned}
\mathbb{C}^{-1} \tilde{\sigma}_{h} & =\tilde{\sigma}_{h} /(2 \mu)-\lambda /(2 \mu)\left(\operatorname{Tr} \tilde{\sigma}_{h}\right) \delta /(2 \lambda+2 \mu) \\
& =\tilde{\mathbb{C}}^{-1} \tilde{\sigma}_{h}-\frac{\varrho}{4} \operatorname{Tr}\left(\sigma_{h}\right) \delta=\tilde{\mathbb{C}}^{-1} \tilde{\sigma}_{h}+\frac{\varrho}{2} p_{h} \delta,
\end{aligned}
$$

and abbreviate

$$
\widehat{\sigma}_{h}:=\tilde{\mathbb{C}}^{-1} \sigma_{h}+\varrho / 2 p_{h} \delta+\gamma_{h} .
$$

The orthogonality in the Helmholtz decomposition (16) leads to

$$
\begin{aligned}
\|\operatorname{Curl} \operatorname{Curl} \Phi\|_{\mathbb{C}^{-1}}^{2}= & \int_{\Omega} \operatorname{Curl} \operatorname{Curl} \Phi:\left(\mathbb{C}^{-1} \tilde{\sigma}_{h}-\varepsilon(w)\right) d x \\
= & \left(\operatorname{Curl} \operatorname{Curl} \Phi, \widehat{\sigma}_{h}\right)_{0, \Omega}-\left(\operatorname{Curl} \operatorname{Curl} \Phi, \varrho / 2\left(p_{h}\right.\right. \\
& \left.\left.\left.+1 / 2 \operatorname{Tr}\left(\sigma_{h}\right)\right)\right) \delta\right)_{0, \Omega}-(\operatorname{Curl} \operatorname{Curl} \Phi, \nabla w)_{0, \Omega}
\end{aligned}
$$

where we have already used the aforementioned notation and the fact that $\operatorname{Curl} \operatorname{Curl} \Phi$ is symmetric (and so orthogonal to asymmetric $\sigma_{h}-\tilde{\sigma}_{h}$ and $\gamma_{h}$ ). Lemma 2 yields

$$
\begin{aligned}
& \|\operatorname{Curl} \operatorname{Curl} \Phi\|_{L}^{2} \lesssim\left(\operatorname{Curl} \operatorname{Curl} \Phi, \widehat{\sigma}_{h}-\nabla w\right)_{0, \Omega} \\
& +\left\|\varrho / 2\left(p_{h}+1 / 2 \operatorname{Tr}\left(\sigma_{h}\right)\right)\right\|_{0, \Omega}\|\operatorname{Curl} \operatorname{Curl} \Phi\|_{L} .
\end{aligned}
$$

The estimation of $\left(\operatorname{Curl} \operatorname{Curl} \Phi, \hat{\sigma}_{h}\right)_{0, \Omega}$ essentially follows the technique of [11, Lemma 5.1]. The first observation is that $b:=\operatorname{Curl} \Phi \in H^{1}(\Omega)^{2}$ is constant on each of the connectivity components $\Gamma_{j}$ of $\Gamma_{N}$. Taking nodal interpolation as a boundary condition on $\Gamma_{N}$ and a Clement-type weak interpolant, e.g. $b_{h}(z):=\left|\omega_{z}\right|^{-1} \int_{\omega_{z}} b(x) d x$ for a node $z \notin \bar{\Gamma}_{N}$ with patch $\omega_{z}:=\operatorname{int}\left(\cup\left\{K \in \mathcal{T}_{h}: z \in K\right\}\right)$ and $b_{h}(z):=b(z)=c_{j}$ for $z \in \bar{\Gamma}_{j}$, one defines $b_{h} \in C(\bar{\Omega})^{2} \cap\left(\mathbb{P}_{1}\left(\mathcal{T}_{h}\right)\right)^{2}$ with $b-b_{h}=0$ on $\Gamma_{N}$ and so is Curl $b n=0=\operatorname{Curl} b_{h} n$ almost everywhere $\Gamma_{N}$. Furthermore,

$$
\left\|h_{\mathcal{T}_{h}}^{-1}\left(b-b_{h}\right)\right\|_{0, \Omega}+\left\|h_{\mathcal{E}_{h}}^{-1 / 2}\left(b-b_{h}\right)\right\|_{0, \mathcal{E}_{h}} \lesssim|b|_{1, \Omega} \lesssim|\Phi|_{2, \Omega} .
$$

Since Curl $b_{h} \in P_{0}\left(\mathcal{T} ; \mathbb{M}^{2 \times 2}\right)$ with div $\operatorname{Curl} b_{h}=0, \tau_{h}:=\operatorname{Curl} b_{h} \in$ $\Sigma_{0, h}$ and (13) 1 yield

$$
\begin{array}{r}
\int_{\Gamma_{D}} u_{D} \cdot \tau_{h} n d s=\left(\tilde{\mathbb{C}}^{-1} \sigma_{h}, \tau_{h}\right)_{0, \Omega}+\left(\gamma_{h}, \tau_{h}\right)_{0, \Omega} \\
+\left(\varrho / 2 p_{h}, \operatorname{Tr} \tau_{h}\right)_{0, \Omega}=\left(\widehat{\sigma}_{h}, \tau_{h}\right)_{0, \Omega} .
\end{array}
$$

This and $\tau_{h} n=0$ along $\Gamma_{N}$ plus an integration by parts show that

$$
\left(\hat{\sigma}_{h}, \tau_{h}\right)_{0, \Omega}=\int_{\Gamma_{D}} w_{D} \cdot \operatorname{Curl} b_{h} n d s=\int_{\Gamma} w \cdot \operatorname{Curl} b_{h} n d s=\left(\operatorname{Curl} b_{h}, \nabla w\right)_{0, \Omega} .
$$


Therefore,

$\left(\operatorname{Curl} \operatorname{Curl} \Phi, \hat{\sigma}_{h}-\nabla w\right)_{0, \Omega}=\left(\operatorname{Curl}\left(b-b_{h}\right), \hat{\sigma}_{h}-\nabla w\right)_{0, \Omega}$.

A standard piecewise integration by parts yields

$$
\begin{aligned}
\left(\operatorname{CurlCurl} \Phi, \hat{\sigma}_{h}-\nabla w\right)_{0, \Omega}= & \left(b-b_{h}, \operatorname{Curl}_{\mathcal{T}_{h}} \hat{\sigma}_{h}\right)_{0, \Omega} \\
& -\int_{\cup \mathcal{E}_{\Omega}}\left(b-b_{h}\right) \cdot J\left(\hat{\sigma}_{h}\right) t_{E} d s \\
& -\int_{\Gamma_{D}}\left(b-b_{h}\right)\left(\hat{\sigma}_{h} t_{E}-\partial u_{D} / \partial s\right) d s .
\end{aligned}
$$

From this, using Cauchy inequalities, trace estimates and (19), we eventually conclude the proof; cf. [11] for further details.

Lemma 4 and inequality (18) show that

$$
\begin{aligned}
\left\|\sigma-\tilde{\sigma}_{h}\right\|_{L}+\left\|u-w-\tilde{u}_{h}\right\|_{V} \lesssim\left\|h_{\mathcal{T}_{h}} \operatorname{Curl}_{\mathcal{T}_{h}}\left(\tilde{\mathbb{C}}^{-1} \sigma_{h}+\varrho / 2 p_{h} \delta+\gamma_{h}\right)\right\|_{0, \Omega} \\
+\left\|h_{\mathcal{E}_{h}}^{1 / 2} J\left(\tilde{\mathbb{C}}^{-1} \sigma_{h}+\varrho / 2 p_{h} \delta+\gamma_{h}\right) t_{E}\right\|_{0, \mathcal{E}_{\Omega} \cup \Gamma_{D}} \\
+\left\|h_{\mathcal{T}_{h}}\left(f+\operatorname{div} \mathcal{T}_{h} \sigma_{h}\right)\right\|_{0, \Omega}+\left\|h_{\mathcal{E}_{h}}^{1 / 2}\left(g_{N}-\sigma_{h} n\right)\right\|_{0, \Gamma_{N}} \\
\quad+\left\|\operatorname{As} \sigma_{h}\right\|_{L}+\left\|\varrho / 2\left(p_{h}+1 / 2 \operatorname{Tr}\left(\sigma_{h}\right)\right)\right\|_{0, \Omega}
\end{aligned}
$$

Lemma 5 There exists a constant $C_{3}>0$ independent of $\lambda$ and $h$, such that

$$
2 \mu\left\|\nabla u-\widehat{\sigma}_{h}\right\|_{0, \Omega} \leq C_{3} \Phi\left(\sigma_{h} ; \mathcal{T}_{h}\right) .
$$

Proof. The proof follows the lines of that in [11, Lemma 5.3] with minor modifications (partly) related to the change of $\mathbb{C}$ into $\tilde{\mathbb{C}}$. The main ingredients are: $i$ ) some Helmholtz decomposition $\nabla u-\widehat{\sigma}_{h}=\tilde{\mathbb{C}}^{-1} \operatorname{Curl} f+\nabla q$ and $\int_{\Omega} \widehat{\sigma}_{h}$ : Curl $R_{h} f d x=0$ (in the notation of [11]); ii) the property $\|\varepsilon(q)\|_{\tilde{\mathbb{C}}} \leq\|\varepsilon(q)\|_{\mathbb{C}}$ at one stage; iii) the use of [11, Lemma 4.1] for estimating $\left\|p-p_{h}\right\|_{0, \Omega}$. Hence, the details are omitted.

Observing that the symmetric part of the stress error $\left\|\sigma-\tilde{\sigma}_{h}\right\|_{L}$ provides a control on the complete stress error $\left\|\sigma-\sigma_{h}\right\|_{L}$ (see [10], Sect. 4.4), combining (20) and Lemma 5 yields the reliability estimate (4).

\section{Efficiency}

Given the reliable error estimator with $\eta_{K}$ and $\eta_{E}$, this section aims to establish the reverse estimate for $\eta_{K}$ and $\eta_{E}$ given by (5) and (6), respectively.

Notice that the last term $\left\|h_{K}\left(f-f_{K}\right)\right\|_{0, K}$ in (5), with the integral mean $f_{K}$ of $f$ on $K$, is an oscillation of the right-hand side and is of higher 
order (provided $f \in H^{1}(K)$ ). The proof of (5) is by a standard inverse estimate technique due to Verfürth [28]. We therefore give an example for $\left\|h_{K}\left(\operatorname{Curl} \hat{\sigma}_{h}\right)\right\|_{0, K}=: \varrho_{1}\left(\hat{\sigma}_{h}\right)$ and the polynomial $\hat{\sigma}_{h}:=\tilde{\mathbb{C}}^{-1} \sigma_{h}+$ $\varrho / 2 p_{h} \delta+\gamma_{h} \in \mathbb{P}_{2}(K)$.

On the finite-dimensional vector space $\mathbb{P}_{2}(K), \varrho_{1}$ is a seminorm and so is $\varrho_{2}$, defined by

$$
\varrho_{2}\left(\hat{\tau}_{h}\right):=\min _{v \in H^{1}(K)}\left\|\hat{\tau}_{h}-\nabla v\right\|_{0, K} \quad \text { for } \hat{\tau}_{h} \in \mathbb{P}_{2}(K) .
$$

Notice that $\varrho_{2}\left(\hat{\tau}_{h}\right)=0$ implies that $\hat{\tau}_{h}$ is a gradient and hence $\varrho_{1}\left(\hat{\tau}_{h}\right)=$ 0 . Owing to the equivalence of norms on finite dimensional vector spaces (here, a quotient space of $\mathbb{P}_{2}(K)$ factorized by the set of gradients) one deduces that $\varrho_{1} \lesssim \varrho_{2}$. A scaling argument reveals that the constant $C$ in $\varrho_{1} \leq C \varrho_{2}$ on $\mathbb{P}_{2}(K)$ is independent of $h_{K}$. Therefore, we have that

$$
\left\|h_{K} \operatorname{Curl} \hat{\sigma}_{h}\right\|_{0, K} \lesssim\left\|\tilde{\mathbb{C}}^{-1}\left(\sigma-\sigma_{h}\right)+\varrho / 2\left(p-p_{h}\right) \delta+\gamma-\gamma_{h}\right\|_{0, K}
$$

and triangle inequalities yield the associated result in (5). The remaining assertions in (5) are easier to prove; we therefore omit further details on $\eta_{K}$.

The interior edge contributions $\eta_{E}$ for $E \in \mathcal{E}_{\Omega}$ satisfy

$$
\begin{aligned}
& h_{E}^{1 / 2}\left\|J\left(\left(\tilde{C}^{-1} \sigma_{h}+\varrho / 2 p_{h} \delta+\gamma_{h}\right) t_{E}\right)\right\|_{0, E} \\
\lesssim & \left\|\sigma-\sigma_{h}\right\|_{0, \omega_{E}}+\left\|p-p_{h}\right\|_{0, \omega_{E}}+\left\|\gamma-\gamma_{h}\right\|_{0, \omega_{E}}+\left\|h_{\mathcal{T}}\left(f-f_{\mathcal{T}}\right)\right\|_{0, \omega_{E}}
\end{aligned}
$$

with a right-hand side that is the sum of the right-hand side in (5) for the two neighbouring elements $K=T_{ \pm}, \bar{\omega}_{E}=T_{+} \cup T_{-}$and $T_{ \pm} \in \mathcal{T}_{h}$ with $T_{+} \cap T_{-}=E$. The proof of (6) follows the lines of that of (5) on the finite dimensional space $\mathbb{P}_{2}\left(T_{+}\right) \oplus \mathbb{P}_{2}\left(T_{-}\right)$of piecewise polynomial functions on $\omega_{E}$ (and $\left\|\hat{\tau}_{h}-\nabla v\right\|_{0, \omega_{E}}$ ) in the definition of $\varrho_{2}$. We therefore omit further details and, instead, mention the standard technique for the proof of (5)-(6) which employs cubic and quadratic bubble-functions and an integration by parts. In this way, one can prove that $\eta_{E}$ is efficient for $E \in \mathcal{E}_{D} \cup \mathcal{E}_{N}$ in the following sense

$$
\begin{aligned}
\eta_{E} & \lesssim h_{E}^{1 / 2}\left\|\sigma_{h} n_{E}-g_{N}\right\|_{0, E} \\
& \lesssim\left\|\sigma-\sigma_{h}\right\|_{0, \omega_{E}}+\left\|h_{E}\left(f-f_{\mathcal{T}_{h}}\right)\right\|_{0, \omega_{E}}+h_{E}^{1 / 2}\left\|g_{N}-g_{N, E}\right\|_{0, E} \quad \forall E \in \mathcal{E}_{N}, \\
\eta_{E} & \lesssim h_{E}^{1 / 2}\left\|\left(\tilde{\mathbb{C}}^{-1} \sigma_{h}+\varrho / 2 p_{h} \delta+\gamma_{h}-\nabla u_{D}\right) t_{E}\right\|_{0, E} \\
& \lesssim h_{E}^{1 / 2}\left\|\left(\tilde{\mathbb{C}}^{-1}+\varrho / 2 p_{h} \delta+\gamma_{h}-\nabla u_{D, E}\right) t_{E}\right\|_{0, E}+h_{E}^{1 / 2}\left\|\partial / \partial s\left(u_{D}-u_{D, E}\right)\right\|_{0, E} \\
& \lesssim\left\|\sigma-\sigma_{h}\right\|_{0, \omega_{E}}+\left\|p-p_{h}\right\|_{0, \omega_{E}}+\left\|\gamma-\gamma_{h}\right\|_{0, \omega_{E}}+h_{E}^{1 / 2}\left|u_{D}-u_{D, E}\right|_{1, E}
\end{aligned}
$$


for all $E \in \mathcal{E}_{D}$.

Therein, $\bar{\omega}_{E}=K \in \mathcal{T}_{h}$ is the neighbouring element of $E \subseteq K \cap \Gamma$ and, at least for smooth data $g_{N}$ and $u_{D}, g_{N, E}$ and $u_{D, E}$ are their constant and affine approximations on $E$, respectively. Further details on the adopted arguments can be found in $[11,12]$ and are therefore omitted here.

\section{Adaptive algorithm}

The efficiency in the local form asserted above motivates the usage of $\Phi_{K}^{2}$ as a refinement indicator in an adaptive mesh-refining algorithm.

The following adaptive Algorithm (A) has been implemented in the framework of a Mat lab computer code:

a. Start with a coarse mesh $\mathcal{T}_{h, 0}, k=0$, and a fixed tolerance tol $>0$.

b. Solve the discrete problem with respect to the present mesh $\mathcal{T}_{h, k}$ with $N$ degrees of freedom.

c. Compute $\Phi_{K}$ for all $K \in \mathcal{T}_{h, k}$ with a sum over all the edges $E$ of $K$.

d. Compute the error estimate

$$
\Phi_{k}=\Phi_{k}\left(\sigma_{h} ; \mathcal{T}_{h, k}\right)=\left(\sum_{K \in \mathcal{T}_{h, k}} \Phi_{K}^{2}\right)^{1 / 2}
$$

corresponding to the mesh $\mathcal{T}_{h, k}$. Then, terminate the algorithm if

$$
\Phi_{k} \leq \operatorname{tol} \Phi_{0},
$$

otherwise go to step (e) of the algorithm.

e. Mark the element $K$ for further refinement if $\Phi_{K} \geq \frac{1}{2} \max _{K^{\prime} \in \mathcal{T}_{h, k}} \Phi_{K^{\prime}}$.

f. Perform red-green-blue refinement on all the marked elements and run a closure algorithm to avoid hanging nodes.

g. Generate the new mesh $\mathcal{T}_{h, k+1}$, set $k \rightarrow k+1$, and go to $(b)$.

Details on the red-green-blue refinement and closure algorithms can be found in $[29,20,8]$. The numerical performance of Algorithm (A) will be illustrated in Section 6 in the solution of several compressible and incompressible benchmark problems with singularities.

\section{Numerical experiments}

To provide experimental evidence of the robustness and accuracy of the DMH method with adaptive mesh refinement and of the reliability, efficiency and robustness of the a-posteriori error estimator, we investigate several model problems, both in the incompressible (Stokes problem) and 
in the compressible regime. We also compare the results provided by algorithm (A) with uniform mesh-refinement. In the following, the parameter $N$ represents the total number of degrees of freedom associated with a certain mesh, while $h$ represents an average mesh size (in the case of uniform refinement).

\subsection{Stokes flow in an L-shaped domain}

The first numerical example deals with the solution of the Stokes problem in the L-shaped domain shown in Fig. 1, with $f=0$ and viscosity $\mu=1$. The boundary values, prescribed as specified in Fig. 1 (left), are computed from the exact solution, which reads as a function of the polar coordinates $(r, \theta)$

$$
\begin{aligned}
& u=r^{\alpha}\left((1+\alpha) \sin (\theta) w(\theta)+\cos (\theta) w_{\theta}(\theta)\right), \\
& v=r^{\alpha}\left(-(1+\alpha) \cos (\theta) w(\theta)+\sin (\theta) w_{\theta}(\theta)\right), \\
& p=-r^{(\alpha-1)}\left((1+\alpha)^{2} w_{\theta}(\theta)+w_{\theta \theta \theta}(\theta)\right) /(1-\alpha),
\end{aligned}
$$

with

$$
\begin{aligned}
w(\theta)= & (\sin ((1+\alpha) \theta) \cos (\alpha \omega)) /(1+\alpha)-\cos ((1+\alpha) \theta) \\
& -(\sin ((1-\alpha) \theta) \cos (\alpha \omega)) /(1-\alpha)+\cos ((1-\alpha) \theta),
\end{aligned}
$$

and where $\alpha=0.54448373$ is the positive solution of the equation $\alpha \sin (2 \omega)+$ $\sin (2 \omega \alpha)=0$, with $\omega=3 \pi / 4$.
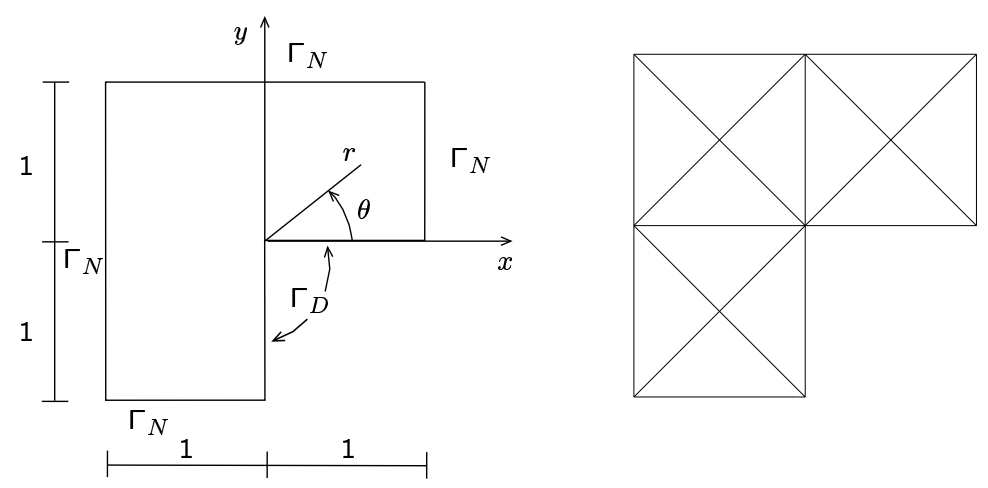

Figure 1. Computational domain (left) and initial mesh (right) for the numerical example of Subsection 6.1.

A plot of the initial mesh is given in Fig. 1 (right), while in Fig. 2 we show the mesh generated by Algorithm (A) after 12 refinement steps and 
a magnified detail of the mesh around the re-entrant corner at $(0,0)$. Notice the strong nonuniformity of the computational mesh and the high refinement performed by the automatic mesh generation algorithm near the singularity.

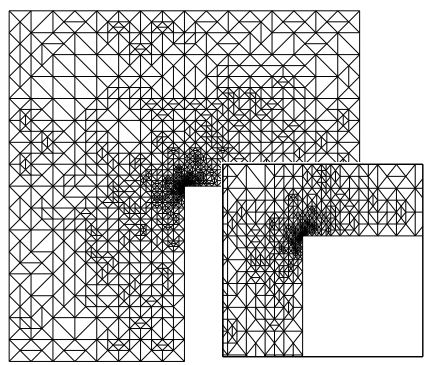

Figure 2. Adaptive mesh generated by Algorithm (A) after 12 refi nement steps with a magnifi ed detail around the re-entrant corner at $(0,0)$ for the numerical example of Subsection 6.1.

The experimental convergence rate for the uniform refinement tends to the theoretically expected rate $\alpha$ which results from the approximation of singular functions like (22) (see [24]). The convergence rate we obtain with the adaptive mesh refinement is improved to the optimal order 1 . These results are shown in Fig. 3, where the error $\left\|\left|\sigma-\sigma_{h}\right|\right\|=\left\|\widetilde{C}^{-1 / 2}\left(\sigma-\sigma_{h}\right)\right\|_{0, \Omega}$ and the error estimator $\Phi\left(\sigma_{h} ; \mathcal{T}_{h}\right)$ are displayed as functions of $N$ on uniform and adaptive meshes (notice that in Fig. 3, a slope 1/2 corresponds to a convergence rate of 1$)$.

In Tab. 1 we show the error $\left\|\left|\sigma-\sigma_{h}\right|\right\|$ and the error $\left\|u-u_{h}\right\|_{0, \Omega}$, both computed using a high-order Gauss quadrature formula on each mesh element.

The experimental convergence rate $C R$ is defined as the corresponding (negative) slope in Fig. 3. We observe that the quotient $\frac{\Phi\left(\sigma_{h} ; \mathcal{T}_{h}\right)}{\left\|\sigma-\sigma_{h}\right\|}$ remains bounded from above in agreement with estimate (4). 


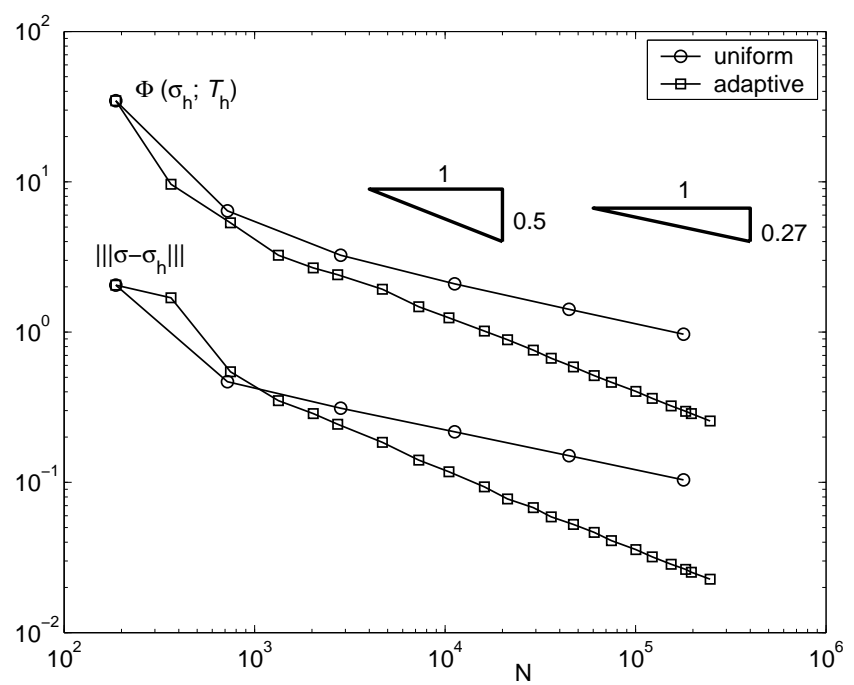

Figure 3. Error and error estimator for the numerical example of Subsection 6.1 on uniform and adaptive meshes.

\begin{tabular}{ccccccc}
\hline$N$ & $h$ & $\left\|\sigma-\sigma_{h}\right\|$ & $\mathrm{CR}$ & $\left\|u-u_{h}\right\|_{0, \Omega}$ & $\mathrm{CR}$ & $\frac{\Phi\left(\sigma_{h} ; \mathcal{T}_{h}\right)}{\left\|\sigma-\sigma_{h}\right\|}$ \\
\hline 187 & 1.4142 & $2.052 \mathrm{e}+00$ & - & $8.728 \mathrm{e}+00$ & - & 0.0592 \\
721 & 0.7071 & $4.673 \mathrm{e}-01$ & 2.135 & $5.643 \mathrm{e}-01$ & 3.951 & 0.0730 \\
2833 & 0.3536 & $3.118 \mathrm{e}-01$ & 0.584 & $1.940 \mathrm{e}-01$ & 1.540 & 0.0961 \\
11233 & 0.1770 & $2.169 \mathrm{e}-01$ & 0.523 & $8.188 \mathrm{e}-02$ & 1.244 & 0.1036 \\
44737 & 0.0884 & $1.505 \mathrm{e}-01$ & 0.527 & $3.716 \mathrm{e}-02$ & 1.139 & 0.1063 \\
178561 & 0.0442 & $1.038 \mathrm{e}-01$ & 0.534 & $1.740 \mathrm{e}-02$ & 1.094 & 0.1075 \\
\hline
\end{tabular}

Table 1. Error and convergence rates on uniform meshes for the numerical example of Subsection 6.1.

In Tab. 2 we show the individual contributions to the error estimator, defined as follows

$$
\begin{aligned}
\eta_{\text {curl }} & =\left(\sum_{K \in \mathcal{T}_{h}}\left\|\operatorname{curl}\left(\widetilde{\mathbb{C}}^{-1} \sigma_{h}+\frac{\rho}{2} p_{h} \delta+\gamma_{h}\right)\right\|_{0, K}^{2}\right)^{1 / 2}, \\
\eta_{\text {As }}= & \left(\sum_{K \in \mathcal{T}_{h}} \frac{1}{\mu^{2}}\left\|\operatorname{As} \sigma_{h}\right\|_{0, K}^{2}\right)^{1 / 2}, \\
\eta_{E}= & \left(\sum_{e \in \mathcal{E}_{\Omega} \cup \mathcal{E}_{D}} h_{E}\left\|J\left(\left(\widetilde{\mathbb{C}}^{-1} \sigma_{h}+\frac{\rho}{2} p_{h} \delta+\gamma_{h}\right) t_{E}\right)\right\|_{0, E}^{2}\right. \\
& \left.+\sum_{e \in \mathcal{E}_{N}} \frac{h_{E}}{\mu^{2}}\left\|\sigma_{h} n_{E}-g_{N}\right\|_{0, E}^{2}\right)^{1 / 2} .
\end{aligned}
$$


The remaining contributions to the error estimator, $\eta_{\text {div }}=\left(\sum_{K \in \mathcal{T}_{h}} \frac{h_{K}^{2}}{\mu^{2}} \| f+\right.$ $\left.\operatorname{div} \sigma_{h} \|_{0, K}^{2}\right)^{1 / 2}$ and $\eta_{T r}=\left(\sum_{K \in \mathcal{T}_{h}}\left\|\frac{\rho}{2}\left(p_{h}+\frac{1}{2} \operatorname{Tr} \sigma_{h}\right)\right\|_{0, K}^{2}\right)^{1 / 2}$, here as well as in the following examples, are of negligible size compared to the other contributions.

\begin{tabular}{cccccccc}
\hline$N$ & $h$ & $\eta_{\text {curl }}$ & $\mathrm{CR}$ & $\eta_{\text {As }}$ & $\mathrm{CR}$ & $\eta_{E}$ & $\mathrm{CR}$ \\
\hline 187 & 1.4142 & $1.939 \mathrm{e}+01$ & - & $1.010 \mathrm{e}+01$ & - & $2.685 \mathrm{e}+01$ & - \\
721 & 0.7071 & $3.405 \mathrm{e}+00$ & 2.509 & $2.071 \mathrm{e}+00$ & 2.286 & $5.001 \mathrm{e}+00$ & 2.424 \\
2833 & 0.3536 & $1.475 \mathrm{e}+00$ & 1.207 & $1.167 \mathrm{e}+00$ & 0.827 & $2.638 \mathrm{e}+00$ & 0.922 \\
11233 & 0.1770 & $9.156 \mathrm{e}-01$ & 0.688 & $7.725 \mathrm{e}-01$ & 0.595 & $1.716 \mathrm{e}+00$ & 0.620 \\
44737 & 0.0884 & $6.156 \mathrm{e}-01$ & 0.572 & $5.238 \mathrm{e}-01$ & 0.560 & $1.161 \mathrm{e}+00$ & 0.563 \\
178561 & 0.0442 & $4.195 \mathrm{e}-01$ & 0.553 & $3.577 \mathrm{e}-01$ & 0.550 & $7.935 \mathrm{e}-01$ & 0.550 \\
\hline
\end{tabular}

Table 2. Individual contributions to the error estimator on uniform meshes.

\subsection{Stokes flow over a backward facing step}

As a next example, we consider a fluid flow through a backward facing step as shown in Fig. 4, with $\mu=1 / 50$. On $\Gamma_{N}$ we set

$$
\begin{array}{ll}
g=(68,(2 y-3) / 1100) & x=0, y \in[1,2], \\
g=(17,(1-y) / 4400) & x=8, y \in[0,2],
\end{array}
$$

while homogeneous boundary conditions are enforced on $\Gamma_{D}$.

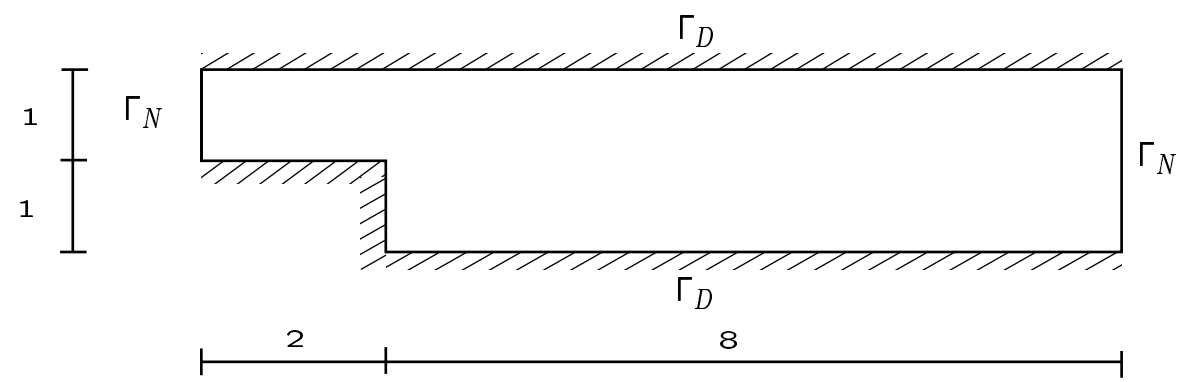

Figure 4. Computational domain and boundary conditions for the numerical example of Subsection 6.2.

In Fig. 5 we show the refined mesh and a magnified detail around the corner after 15 refinement steps with Algorithm (A). The adaptive mesh is highly refined in correspondence of the areas of strongest stretching and curvature of the flow, in particular around the step corner. In Fig. 6 we show 
the error estimator $\Phi\left(\sigma_{h} ; \mathcal{T}_{h}\right)$ as a function of $N$ for adaptive and uniform meshes. The convergence rate is slightly more than $1 / 2$ for adaptive meshes and about $2 / 5$ for uniform meshes.

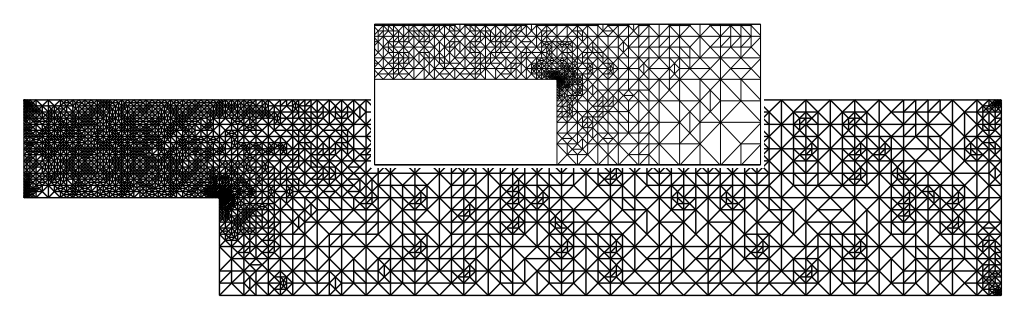

Figure 5. Adapted mesh after 15 iterations of Algorithm (A) and magnifi ed detail around the corner for the numerical example of Subsection 6.2.

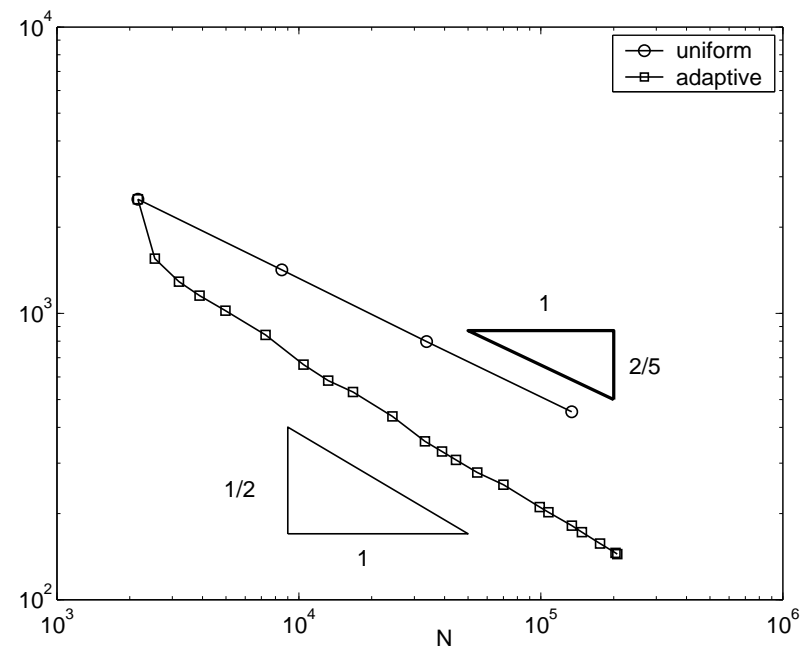

Figure 6. Error estimator as a function of the number of degrees of freedom for uniform and adaptive refi nement for the numerical example of Subsection 6.2. 


\subsection{L-shaped compressible material domain}

We now apply the adaptive refinement algorithm (A) to the numerical solution of the linear elasticity system (7) in both compressible and quasi incompressible regimes. With this aim, we consider an L-shaped domain as in Fig. 7. The radial and tangential components of the exact solution expressed as functions of the polar coordinates $(r, \theta)$ read

$$
\begin{aligned}
& u_{r}(r, \theta)=\frac{r^{\alpha}}{2 \mu}\left(-(\alpha+1) \cos ((\alpha+1) \theta)+\left(C_{2}-(\alpha+1)\right) C_{1} \cos ((\alpha-1) \theta)\right), \\
& u_{\theta}(r, \theta)=\frac{r^{\alpha}}{2 \mu}\left((\alpha+1) \sin ((\alpha+1) \theta)+\left(C_{2}+\alpha-1\right) C_{1} \sin ((\alpha-1) \theta)\right),
\end{aligned}
$$

where $\alpha$ is the same value as in test case $6.1, \omega=3 \pi / 4$ and

$$
C_{1}=-\cos ((\alpha+1) \omega) / \cos ((\alpha-1) \omega), \quad C_{2}=2(\lambda+2 \mu) /(\lambda+\mu) .
$$

The Young modulus is $E=100000$ and numerical computations have been performed with the Poisson ratio $\nu$ ranging in the interval

$$
\nu=[0.3,0.45,0.49,0.499,0.4999,0.49999],
$$

the latter value corresponding to a quasi-incompressible problem. Mixed Dirichlet-Neumann boundary conditions are computed from the exact solution and enforced as shown in Fig. 7.
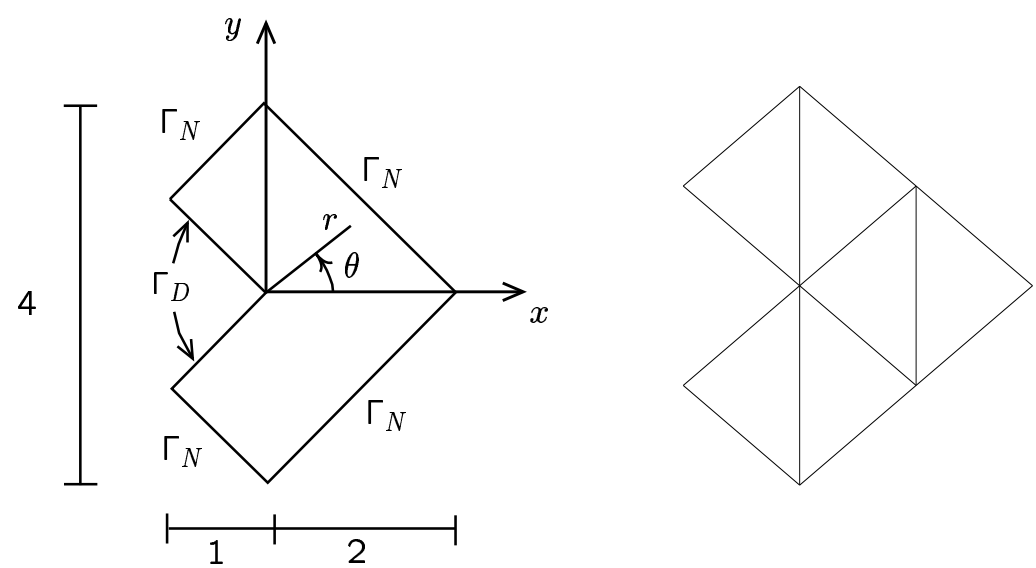

Figure 7. Computational domain (left) and initial mesh (right) for the numerical example of Subsection 6.3.

In Tab. 3 and Tab. 4 the errors and rates of convergence are displayed for $\nu=0.3$ and $\nu=0.49999$, respectively. The experimental convergence rate 
tends to the value $\alpha$, as theoretically expected for solutions like (23)(see [24]). The quotient $\frac{\left\|\sigma-\sigma_{h}\right\| \|}{\Phi\left(\sigma_{h} ; \mathcal{T}_{h}\right)}$ is seen to be bounded from above and below. In Fig. 8 and Tab. 5 we summarize the results of the computation with Algorithm (A). The final mesh after 20 refinement steps is shown in Fig. 9 (left) with von Mises stresses (right). A very strong refinement is produced by the algorithm around the re-entrant corner.

\begin{tabular}{ccccccc}
\hline$N$ & $h$ & $\left\|\sigma-\sigma_{h} \mid\right\|$ & $\mathrm{CR}$ & $\left\|u-u_{h}\right\|_{2, \Omega}$ & $\mathrm{CR}$ & $\frac{\left\|\sigma-\sigma_{h}\right\|}{\Phi\left(\sigma_{h} ; \mathcal{T}_{h}\right)}$ \\
\hline 100 & 1.4142 & $2.247 \mathrm{e}-02$ & - & $6.487 \mathrm{e}-04$ & - & 42.719 \\
373 & 0.7071 & $9.512 \mathrm{e}-03$ & 1.240 & $9.341 \mathrm{e}-05$ & 2.796 & 71.241 \\
1441 & 0.3535 & $6.015 \mathrm{e}-03$ & 0.661 & $2.958 \mathrm{e}-05$ & 1.658 & 86.312 \\
5665 & 0.1768 & $4.051 \mathrm{e}-03$ & 0.570 & $1.338 \mathrm{e}-05$ & 1.144 & 89.924 \\
22465 & 0.0884 & $2.758 \mathrm{e}-03$ & 0.554 & $6.651 \mathrm{e}-06$ & 1.008 & 90.986 \\
89473 & 0.0442 & $1.884 \mathrm{e}-03$ & 0.549 & $3.465 \mathrm{e}-06$ & 0.940 & 91.538 \\
\hline
\end{tabular}

Table 3. Error and convergence rates on uniform meshes for the numerical example of Subsection 6.3 with $\nu=0.3$.

\begin{tabular}{ccccccc}
\hline$N$ & $h$ & $\left\|\sigma-\sigma_{h} \mid\right\|$ & $\mathrm{CR}$ & $\left\|u-u_{h}\right\|_{0, \Omega}$ & $\mathrm{CR}$ & $\frac{\left\|\sigma-\sigma_{h}\right\|}{\Phi\left(\sigma_{h} ; \mathcal{T}_{h}\right)}$ \\
\hline 100 & 1.4142 & $2.413 \mathrm{e}-02$ & - & $7.797 \mathrm{e}-04$ & - & 40.191 \\
373 & 0.7071 & $1.014 \mathrm{e}-02$ & 1.250 & $9.371 \mathrm{e}-05$ & 3.056 & 77.237 \\
1441 & 0.3535 & $6.358 \mathrm{e}-03$ & 0.674 & $2.869 \mathrm{e}-05$ & 1.707 & 99.195 \\
5665 & 0.1768 & $4.269 \mathrm{e}-03$ & 0.574 & $1.263 \mathrm{e}-05$ & 1.182 & 104.213 \\
22465 & 0.0884 & $2.904 \mathrm{e}-03$ & 0.555 & $6.114 \mathrm{e}-06$ & 1.047 & 105.636 \\
89473 & 0.0442 & $1.983 \mathrm{e}-03$ & 0.550 & $3.112 \mathrm{e}-06$ & 0.974 & 106.359 \\
\hline
\end{tabular}

Table 4. Error and convergence rates on uniform meshes for the numerical example of Subsection 6.3 with $\nu=0.49999$.

\begin{tabular}{cccccccc}
\hline $\mathrm{N}$ & $\mathrm{h}$ & $\eta_{\text {curl }}$ & $\mathrm{CR}$ & $\eta_{\text {As }}$ & $\mathrm{CR}$ & $\eta_{E}$ & $\mathrm{CR}$ \\
\hline 100 & 1.414214 & $3.229 \mathrm{e}-04$ & - & $8.181 \mathrm{e}-05$ & - & $4.066 \mathrm{e}-04$ & - \\
373 & 0.707107 & $7.228 \mathrm{e}-05$ & 2.159 & $3.254 \mathrm{e}-05$ & 1.330 & $1.073 \mathrm{e}-04$ & 1.920 \\
1441 & 0.353553 & $3.351 \mathrm{e}-05$ & 1.108 & $2.004 \mathrm{e}-05$ & 0.699 & $5.770 \mathrm{e}-05$ & 0.896 \\
5665 & 0.176777 & $2.105 \mathrm{e}-05$ & 0.670 & $1.325 \mathrm{e}-05$ & 0.596 & $3.755 \mathrm{e}-05$ & 0.619 \\
22465 & 0.088388 & $1.406 \mathrm{e}-05$ & 0.581 & $8.923 \mathrm{e}-06$ & 0.570 & $2.531 \mathrm{e}-05$ & 0.568 \\
89473 & 0.044194 & $9.518 \mathrm{e}-06$ & 0.563 & $6.063 \mathrm{e}-06$ & 0.557 & $1.720 \mathrm{e}-05$ & 0.557 \\
\hline
\end{tabular}

Table 5. Individual contributions to the error estimator on uniform meshes for the numerical example of Subsection 6.3 with $\nu=0.3$. 


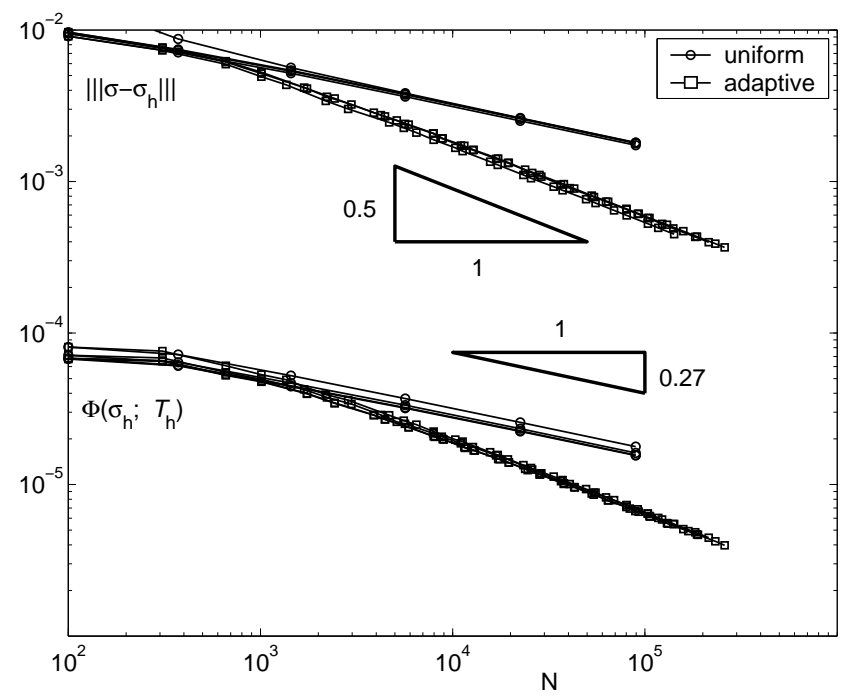

Figure 8. Error and error estimator for uniform and adaptive mesh refi nement for the numerical example of Subsection 6.3 with $\nu=[0.3,0.45,0.49,0.499,0.4999,0.49999]$.
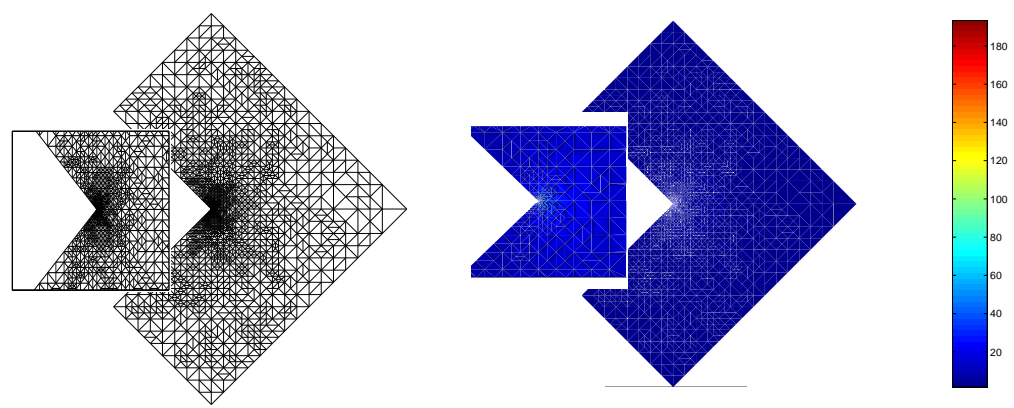

Figure 9. Adapted mesh (left) with a magnifi ed detail of the re-entrant corner and vonMises stresses (right) with a magnifi ed detail of the re-entrant corner after 20 refi nement steps with Algorithm (A) with $\nu=0.3$ for the numerical example of Subsection 6.3.

\subsection{Plate with a hole under traction}

As a final test case, we consider a plate with a circular hole, subject to a shearing load on the right side (see Fig. 10, left).

A closed-form solution to the linear elasticity system exists in the case of an infinitely large, thin plate with a circular hole, and the stress normal to the vertical plane of symmetry at point $P$ (see Fig. 10) is $\sigma_{x x}=3 \sigma_{0}$ 

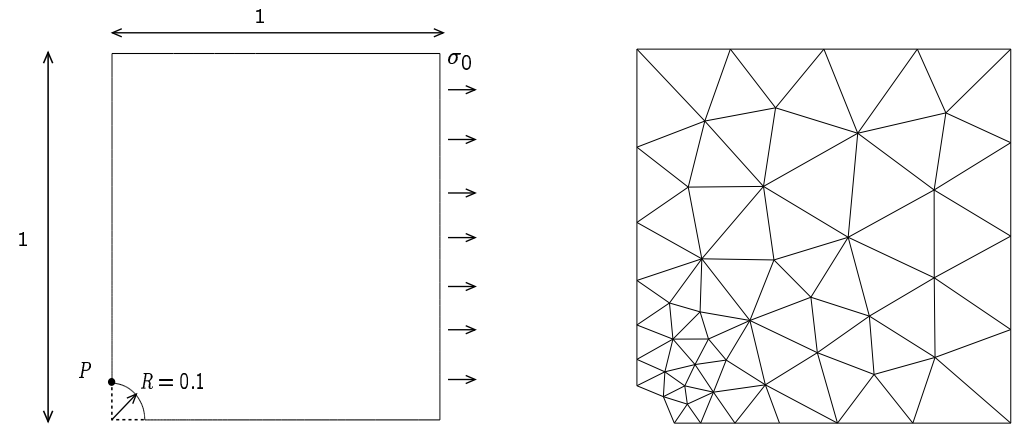

Figure 10. Computational domain (left) and initial mesh (right) for the numerical example of Subsection 6.4.

([30], Eq. (36), with $r=a$ and $\theta=\pi / 2$ ). We set $\sigma_{0}=1, E=100000$ and $\nu=0.3$. Symmetry boundary conditions are enforced on the boundaries converging to the curved part (that is, on the axis of symmetry of the entire plate, of which Fig. 10 represents the quarter that we study), while Neumann boundary conditions are enforced elsewhere. Since point $P$ is a node, we display the value of $\sigma_{x x}$ computed by an averaged stress approximation. Observe the significantly better result obtained with the adaptive approximation. In Fig. 11 we plot the value $\sigma_{x x}$ at $P$ computed using both uniform and adapted meshes. In Fig. 12 (left) we plot the adapted mesh after 15 refinement steps with Algorithm (A) and in Fig. 12 (right) we plot the corresponding von Mises stresses. We observe that the Algorithm (A) generates a refinement towards the hole, where the stress gradients are higher. The results are independent of the Poisson ratio $\nu$. 


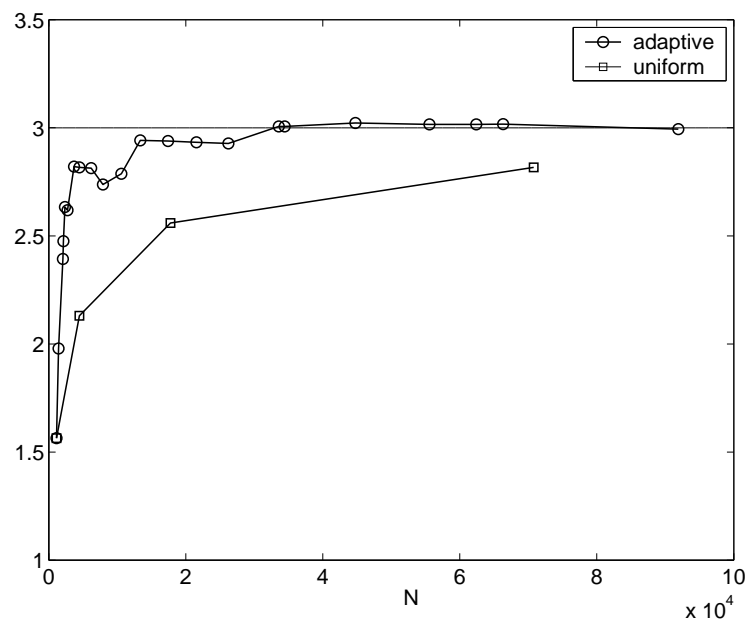

Figure 11. $\sigma_{x x}$ component of the stress tensor at $P$ as a function of the number of degrees of freedom for the numerical example of Subsection 6.4 using uniform and adaptive refi nement.
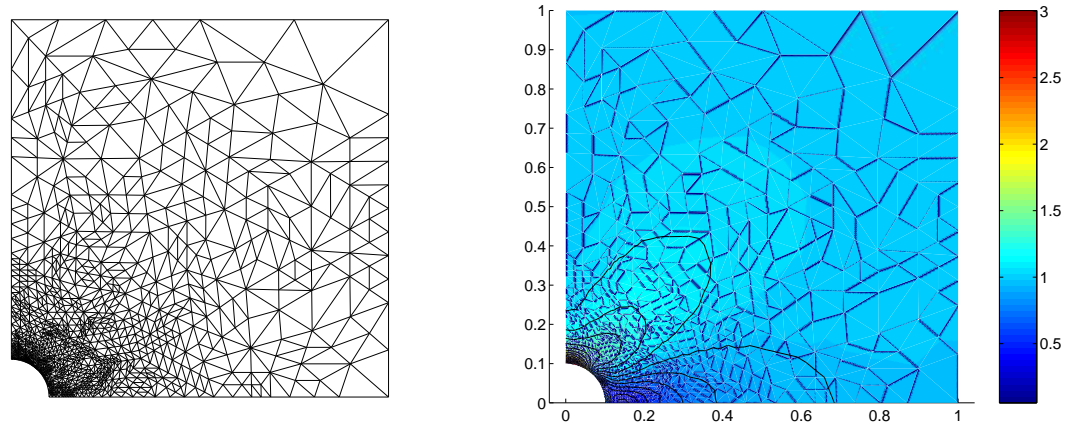

Figure 12. Adapted mesh (left) and von Mises stresses (right) after 15 refi nement steps with Algorithm (A) for the numerical example of Subsection 6.4.

Acknowledgements. Part of this work was done while the first author was visiting the Isaac Newton for Mathematical Sciences, Cambridge, UK. The first author was supported by FWF, P15274 and P16461, DFG Research Center Matheon 'Mathematics for key technologies", C13, EPSRC, N09176/01. The second author was supported by the RTN European Project HaeModel, contract HPRN-CT-2002-00270. The third author was supported by M.U.R.S.T. Cofin 2001 Grant 'Metodi Numerici Avanzati per Equazioni Parziali di Interesse Applicativo". 


\section{References}

1. D.N. Arnold and F. Brezzi. Mixed and nonconforming fi nite element methods: Implementation, postprocessing and error estimates. Math. Modeling and Numer. Anal., 19-1:7-32, 1985.

2. D.N. Arnold, F. Brezzi, and J. Douglas. PEERS: A new mixed fi nite element for plane elasticity. Japan J. Appl. Math., 1:347-367, 1984.

3. D.N. Arnold and R. Falk. A new mixed formulation for elasticity. Numer. Math., 53:13-30, 1988.

4. R.E. Bank and B.D. Welfert. A posteriori error estimates for the Stokes problem. SIAM J. Numer. Anal., 28:591-623, 1991.

5. M.A. Barrientos, G.N. Gatica, and E.P. Stephan. A mixed fi nite method for nonlinear elasticity: Two-fold saddle point approach and a-posteriori error estimate. $\mathrm{Nu}$ mer.Math., 91(2):197-222, 2002.

6. D. Braess. Finite Elements. Cambridge University Press, Cambridge, 2001.

7. D. Braess, C. Carstensen, and B.D. Reddy. Uniform convergence and a posteriori error estimators for the enhanced strain. Numer. Math., 96:461-479, 2004.

8. S. Brenner and C. Carstensen. Finite element methods. Encyclopedia of Computational Mechanics, 2004 (in press).

9. F. Brezzi and M. Fortin. Mixed and Hybrid Finite Element Methods. Springer Verlag, New York, 1991.

10. C. Carstensen. A Unifying Theory of A Posteriori Finite Element Error Control. 2004 (in preparation).

11. C. Carstensen and G. Dolzmann. A posteriori error estimates for mixed FEM in elasticity. Numer. Math., 81:187-209, 1998.

12. C. Carstensen, G. Dolzmann, S.A. Funken, and D.S. Helm. Locking-free adaptive mixed fi nite element methods in linear elasticity. Comput. Methods Appl. Mech. Engrg., 190:1701-1718, 2000.

13. C. Carstensen and S. Funken. Averaging technique for FE-a posteriori error control in elasticity. Part I: Conforming Fem, part II: $\lambda$-independent estimates, part III: Lockingfree nonconforming fem. Comput. Methods Appl. Mech. Engrg, 190:2483-2498, 2001.

14. C. Carstensen and S. Funken. A posteriori error control in low order fi nite element discretizations of incompressible stationary fbw problems. Math. Comp., 70-236:13531381, 2001.

15. P. Causin. Mixed-hybrid Galerkin and Petrov-Galerkin fi nite element formulations in fuid mechanics. PhD thesis, Università degli Studi di Milano, 2002.

16. P. Causin and R. Sacco. A dual-mixed hybrid formulation for flid mechanics problems: Mathematical analysis and application to semiconductor process technology. Comput. Methods Appl. Mech. Engrg., 192:593-612, 2003.

17. P.G. Ciarlet. The Finite Element Method for Elliptic Problems. North Holland, Amsterdam, 1978.

18. B. Cockburn and J. Gopalakrishnan. Error analysis of variable degree mixed methods for elliptic problems via hybridization. to appear in Math.Comp.

19. B. Cockburn and J. Gopalakrishnan. A characterization of hybridized mixed methods for the Dirichlet problem. SIAM J. Numer. Anal., 42:283-301, 2004.

20. K. Eriksson, D. Estep, P. Hansbo, and C. Johnson. Introduction to adaptive methods for differential equations. Acta Numerica, pages 105-158, 1995.

21. M. Farhloul and M. Fortin. Dual hybrid methods for the elasticity and the Stokes problems: a unifi ed approach. Numer. Math., 76-4:419-440, 1997. 
22. G.N. Gatica, N. Heuer, and E.P. Stephan. An implicit-explicit residual error estimator for the coupling of dual-mixed fi nite elements and boundary elements in elastostatics. Math. Meth. in Appl. Sciences, 24(3):179-191, 2001.

23. G.N. Gatica and E.P. Stephan. A mixed-fem formulation for nonlinear incompressible elasticity in the plane. Numer. Meth. Part. Diff. Eqtns., 18(1):105-128, 2002.

24. P. Grisvard. Singularities in elasticity theory. In P.G. Ciarlet and E. Sanchez-Palencia, editors, Applications of multiple scaling in Mechanics, pages 134-150. Masson, Paris, 1987.

25. L.R. Herrmann. Elasticity equations for incompressible and nearly-incompressible materials by a variational theorem. AIAA Jnl., 3-10:1896-1900, 1965.

26. T.J.R. Hughes. The Finite Element Method, Linear Static and Dynamic Analysis. Prentice-Hall, Englewood Cliffs, New Jersey, 1987.

27. P.A. Raviart and J.M. Thomas. A mixed fi nite element method for second order elliptic problems. In I. Galligani and E. Magenes, editors, Mathematical Aspects of Finite Element Methods,I. Springer-Verlag, Berlin, 1977.

28. R. Verfürth. A posteriori error estimator for the Stokes equations. Numer.Math., 55:309-325, 1989.

29. R. Verfürth. A posteriori error estimator for the Stokes equations. II: Nonconforming discretizations. Numer.Math., 60:235-249, 1991.

30. O.C. Zienkiewicz and J.Z. Zhu. The superconvergent patch recovery (SPR) and adaptive fi nite element refi nement. Comput. Methods Appl. Mech. Eng., 101(1-3):207-224, 1992. 